\title{
Transition in circular Couette flow
}

\author{
By DONALD COLES \\ Harvard University, Cambridge, Massachusetts $\dagger$
}

(Received 12 June 1964)

Two distinct kinds of transition have been identified in Couette flow between concentric rotating cylinders. The first, which will be called transition by spectral evolution, is characteristic of the motion when the inner cylinder has a larger angular velocity than the outer one. As the speed increases, a succession of secondary modes is excited; the first is the Taylor motion (periodic in the axial direction), and the second is a pattern of travelling waves (periodic in the circumferential direction). Higher modes correspond to harmonics of the two fundamental frequencies of the doubly-periodic flow. This kind of transition may be viewed as a cascade process in which energy is transferred by non-linear interactions through a discrete spectrum to progressively higher frequencies in a two-dimensional wave-number space. At sufficiently large Reynolds numbers the discrete spectrum changes gradually and reversibly to a continuous one by broadening of the initially sharp spectral lines.

These periodic flows are not uniquely determined by the Reynolds number. For the case of the inner cylinder rotating and the outer cylinder at rest, as many as 20 or 25 different states (each state being defined by the number of Taylor cells and the number of tangential waves) have been observed at a given speed. As the speed changes, theso states replace each other in a repeatable but irreversible pattern of transitions; vortices appear or disappear in pairs, and waves are added or subtracted. More than $\mathbf{7 0}$ such transitions have been found in the speed range up to about 10 times the first critical speed. Regardless of the state, however, the angular velocity of the tangential waves is nearly constant at 0.34 times the angular velocity of the inner cylinder.

The second kind of transition, which will be called catastrophic transition, is characteristic of the motion when the outer cylinder has a larger angular velocity than the inner one. At a fixed Reynolds number, the fluid is divided into distinct regions of laminar and turbulent flow, and these regions are separated by interfacial surfaces which may be propagating in either direction. Under some conditions the turbulent regions may appear and disappear in a random way; under other conditions they may form quite regular patterns. One common pattern of particular interest is a spiral band of turbulence which rotates at very nearly the mean angular velocity of the two walls without any change in shape except possibly an occasional shift from a right-hand to a left-hand pattern. One example of this spiral turbulence is being studied in some detail in an attempt to clarify the role played in transition by interfaces and intermittency.

$\dagger$ On leave of absence from Graduate Aeronautical Laboratories, California Institute of Technology, Pasadena, California. 


\section{Introduction}

Prior to the beginnings of modern experimental research in fluid mechanics, the steady motion of a viscous fluid between concentric rotating cylinders was thought to be a simple linear combination of (1) rotation like a solid body and (2) circulation like a potential vortex, the relative proportion of each being determined by the angular velocities assigned to the two cylindrical walls. Such a flow is now commonly referred to as Couette flow, just as the flow in a circular pipe is commonly referred to as Poiseuille flow, and for the same reason. The primary object of these early experimenters was to test the validity of the Newtonian stress approximation in the Navier-Stokes equations. The method was first to verify the existence of the hypothetical fluid property called the viscosity, using the scaling laws for the known laminar solutions, and then to determine its value, using the solutions in detail. Except for certain difficulties arising from the finite geometry of the experiment as compared to the theoretical model, the test in each case was successful provided only that the flow velocities were sufficiently small.

These same early experiments, which included work by Hagen, Reynolds, and Mallock, also revealed that the elementary laminar flow was always replaced at sufficiently large velocities by some more complicated eddying flow. In particular, the experiments with concentric cylinders by Couette (1890) and by Mallock $(1888,1896)$ indicated that the critical speed was very much lower when the inner cylinder was rotating and the outer cylinder was at rest than vice versa. More or less independently of these researches, the problem of instability in rotating fluids had also attracted attention because of its importance in meteorology, and a simple criterion for inertial instability was worked out by Rayleigh (1916). According to this criterion, an inviscid circulatory flow is unstable whenever the sense of the local rotation (the vorticity) is opposite to the sense of the overall rotation (the angular velocity). $\dagger$ Applied to the cylinder problem, the criterion states that the potential flow is neutrally stable, as is the flow with the inner cylinder at rest, and that the region of instability includes all flows for which the two cylinders rotate in opposite directions.

In a brilliant contribution to the literature of fluid mechanics, Taylor (1923) improved this stability criterion for Couette flow to take account of viscosity, verified his calculations experimentally, and described the secondary flow which appears after the first onset of instability. This secondary flow consists of a regular cellular vortex structure in which closed ring vortices alternating in sign are wrapped around the axis of rotation. To a good approximation, the secondary vorticity is confined to the part of the fluid where there is instability according to the inviscid criterion. If the cylinders are rotating in the same direction, this is the whole of the fluid, but if they are rotating in opposite directions, it is only the region between the inner cylinder and the surface of vanishing tangential velocity.

$\uparrow$ The form usually given to Rayleigh's criterion is that the flow is unstable if the square of the circulation decreases outwards; i.e. if $d r^{2} v^{2} / d r<0$. Inasmuch as $v / r$ is the angular velocity $\omega$, and $d r v / r d r$ is the axial vorticity $\zeta$, the condition $\omega \zeta<0$ stated in the text is an entirely equivalent form of the criterion. 
Because only a very dim light is shed on the physical mechanism of Taylor instability by the Rayleigh criterion, it is worth pointing out that the heart of this mechanism is a kind of gyroscopic precession in the fluid. Suppose that the tangential velocity is initially non-uniform in the axial direction, so that the fluid is locally rotating about a line which is not quite parallel to the cylinder axis. The radial pressure gradient and the centripetal accelerations arising from the curvature of the main flow field are also non-uniform, and exert a net torque (about a tangent) causing the axis of the local vorticity to precess into the tangential direction. The precession generates radial velocities in a meridional plane, and these in turn are coupled to tangential Coriolis accelerations which

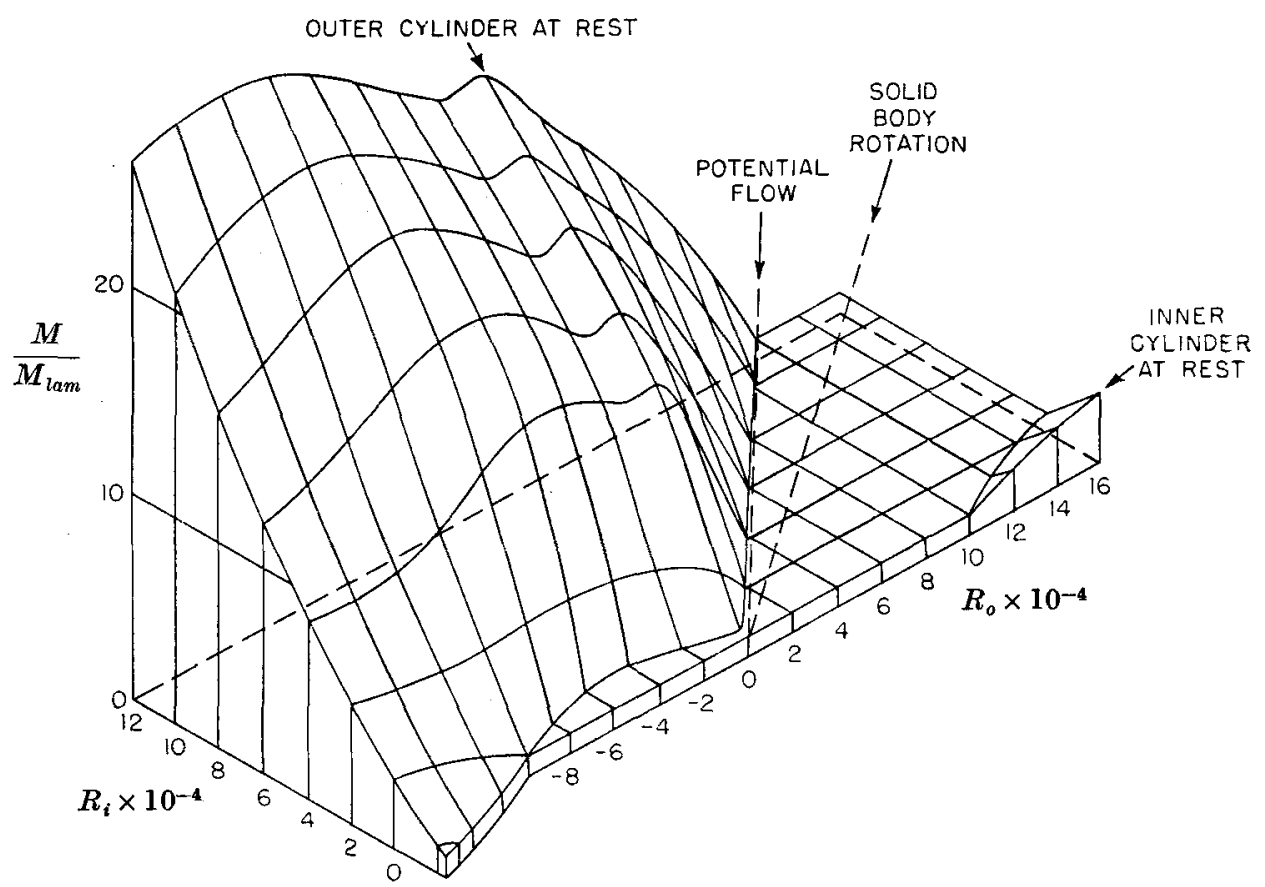

Figure 1. Laminar and turbulent régimes in circular Couette flow according to Wendt (1933, Abb. $\left.9 b ; r_{o} / r_{i}=1 \cdot 176\right)$. Height of surface indicates ratio of measured torque to theoretical laminar torque. Reynolds numbers $R_{i}=\omega_{i} r_{i}^{2} / \nu$ and $R_{o}=\omega_{o} r_{o}^{2} / \nu$ are besed on the radii and surface speeds of the inner and outer cylinders, respectively.

maintain the non-uniformity of the tangential velocity, thereby closing the circle. This description confirms that this type of instability cannot occur if either the vorticity or the streamline curvature is zero in the basic flow. It also shows how viscosity can stabilize the motion by providing tangential viscous forces to balance the Coriolis accelerations and by providing a diffusive mechanism to compensate the generation of tangential vorticity by precession.

Linearized stability analysis is a game which any number can play, and the problem of Taylor instability has by now given rise to a quite extensive analytical literature. Experimental work on transition in Couette flow, on the other hand, has moved at a somewhat slower pace. The researches published up to the beginning of the present investigation, in 1955, tended for the most part to cover 
the same limited ground explored in the earliest work, using flow visualization to determine the Taylor boundary or using torque (sometimes mean-velocity) data to study flow conditions with one or the other cylinder at rest. A useful exception is the investigation by Wendt (1933), who obtained torque data for more general combinations of speed for the two cylinders. Wendt's results for the case $r_{o} / r_{i}=1 \cdot 176$ have been used to construct figure 1. Except for a lack of detail near the transition boundary, and except for mild reservations about the reality of the observed decrease in torque to the left of the ray $R_{o}=0$, the figure provides a satisfactory overall view of the transition problem. In particular, the figure emphasizes the question of the flow behaviour along the ray $R_{i}=0$, where Taylor instability does not occur, and where a hysteresis in the transition was noticed by both Mallock and Taylor (1936). Finally, a valuable contribution to experimental knowledge of the problem was made by Pai (1943), who found for the case of the outer cylinder at rest that the cellular structure persisted far into the turbulent régime, with a hysteresis in the dependence of the flow structure on speed for this case as well.

\subsection{The present research}

The present experimental investigation of stability and transition in Couette flow was begun in 1955 with the object of exploring a number of questions raised by previous work. Interest was initially centred in the problem of the origin of turbulence in the absence of Taylor instability. It was supposed, by analogy with the problem of boundary-layer transition, that a secondary flow periodic in the circumferential direction, with a motion like that of rollers in a roller bearing, might occur as a result of an instability to infinitesimal disturbances. This conjecture is now known on both analytical and experimental grounds to be entirely mistaken. It was with this problem in mind, nevertheless, that it was decided to make the experimental apparatus large enough to permit detailed hot-wire measurements in air. It was also intended that quantitative observations in this apparatus would concentrate on the case of one cylinder rotating and the other at rest. However, the limitations of this approach became apparent as soon as preliminary hot-wire observations had shown the complicated nature of the transition process. With the inner cylinder rotating, the appearance of turbulence was preceded by regular low-frequency oscillatory motions like those described by Taylor (1923). With the outer cylinder rotating, on the other hand, patterns of alternating laminar and turbulent flow were observed. At some speeds these patterns were quite irregular, even random. At other speeds they showed a highly regular alternation at about half the frequency of rotation of the outer cylinder (this was the first observation of the phenomenon now called spiral turbulence). Finally, the expected strong hysteresis was found for the transition in the case of the outer cylinder rotating. When the cylinder speed was slowly increased from zero, the flow remained laminar and steady up to a relatively high speed, at which intermittent turbulence appeared with almost explosive suddenness. Once this transition had occurred, the speed had to be decreased to a fraction of the original value before the turbulence would die out completely and permanently. 
Following these preliminary measurements, the research remained for a time at top dead centre, except for continuing work on the problem of hysteresis. The tripping effect of several different finite disturbances, for example, was studied at some length. The principle obstacle to progress was the lack of a real point of view in the research, combined with the prospect that there would inevitably be a great deal of lost motion if further exploration of the various new phenomena had to depend on tedious hot-wire methods alone.

This impasse was broken in 1957, after a visit to Pasadena by Prof. SchultzGrunow. Using a small portable Couette-flow apparatus, $\dagger$ he demonstrated a beautiful flow-visualization technique making use of suspended metallic particles. A second apparatus was immediately constructed at GALCIT to exploit this method of flow visualization, and the technique has been of immense value in illuminating many details of transition in Couette flow. In early 1958 some of the more interesting phenomena observed in this second machine were recorded in a short motion picture. $f$ During this period, also, several boundaries separating different flow régimes were identified and located quantitatively, although not with utmost precision. The results are shown in figures $2(a),(b),(c)$, which all employ the same co-ordinates as figure 1 but which emphasize different ranges of Reynolds number in anticipation of more specialized discussions later on. The letters A, B, C... scattered about in figure 2 mark the operating points at which certain of the photographs in figures 14-22 (plates 1-12), were taken. The nature of the changes in flow structure which occur at the various boundaries will be made clear in $\S \S 4$ and 5 of the present paper.

At first the small Couette-flow apparatus was expected to yield only qualitative data, except in the matter of the boundaries between different régimes of flow. However, it was soon realized that the suspended-particle technique could also yield quantitative results when combined with suitable optical instrumentation. In order to make the two pieces of experimental apparatus more complementary over the whole range of variables, therefore, a number of changes were undertaken. These included the addition of torque-measuring devices to the small machine; the development of a light source for making shadowgraph pictures of the flow; the replacement of the original glass cylinders by others of greater precision; the provision of a second motor and transmission for the large machine; and the design and fabrication of movable end closures and remotely operated probe-traversing gear for this machine as well. Most of these modifications are described in more detail in $\$ \S 2$ and 3 , which deal with experimental equipment and instrumentation. First, however, a brief indication should be given of the principal results of the experiments carried out during the period 1958-61.

One result which emerged almost immediately from the research was the recognition of two quite different processes of transition from laminar to turbulent

† Some photographs of the flow in this apparatus have been published by SchultzGrunow \& Hein (1956).

$¥$ This motion picture is listed as No. C-2, 'Transition in Circular Couette Flow', in the catalogue of the ASME-ESL Film Library. A copy of the film may be borrowed from the Engineering Societies Library, 345 E. 47th St, New York 17, N.Y., for a nominal fee ( $\$ 5.00$ at the time of writing). 
flow. The first process, which will be called catastrophic transition, is typical of Couette flows dominated by rotation of the outer cylinder (the region to the left of the origin of co-ordinates in figures $2(\mathrm{a})$ and (b)) and is characterized by the familiar phenomenon of intermittency. The flow divides itself into finite regions
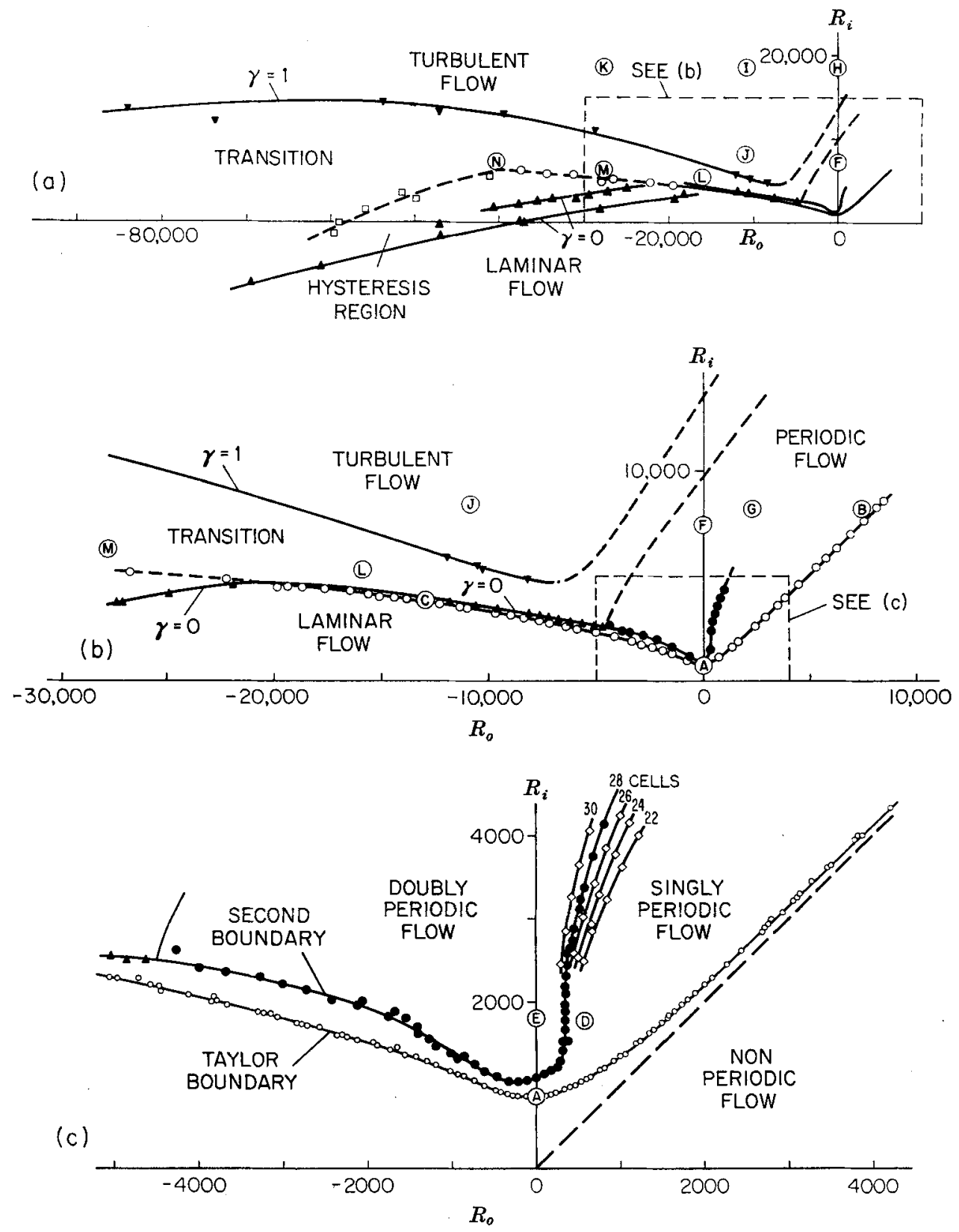

Figure 2. Different régimes in circular Couette flow from visual observations with the small apparatus (rough cylinders; $r_{o} / r_{i}=1 \cdot 135$ ). Reynolds numbers $R_{o}, R_{i}$ are based on radius and surface speed. Data in (b) and (c) are for $v=0.11 \mathrm{~cm}^{2} / \mathrm{sec}$; otherwise $\nu=0.01 \mathrm{~cm}^{2} / \mathrm{sec}$. Circled letters refer to photographs in plates $2,3,4,5$ and 12 . 
which are recognizably either laminar or turbulent and which are separated by quite well-defined interfaces. These interfaces may be propagating in either direction, i.e. into the laminar or into the turbulent regions, and the pattern of mixed laminar-turbulent flow may itself be persistent or non-persistent, random or regular, depending on the particular combination of speeds for the two cylinders.

Probably the most challenging problem in connexion with catastrophic transition is to determine the factors which control the direction and velocity of propagation of laminar-turbulent interfaces. Study of this problem in the case of Couette flow is aided by the fact that a special configuration of mixed flow, a configuration which will be called spiral turbulence, occurs quite generally throughout the transition region. In a typical flow (of. figure 22, plate 12), a turbulent strip and a laminar strip are wrapped around the cylinders in a spiral pattern much like the pattern of stripes on a barber's pole. This spiral pattern rotates at very nearly the mean angular velocity of the two cylinders, without any significant change in size or shape. As a result, the flow observed from either wall shows an astonishing regularity of alternation between laminar and turbulent characteristics, a regularity which has recently been taken as a basis for an elaborate and ambitious investigation of spiral turbulence in the large machine. This investigation will be reported separately, and the further discussion of catastrophic transition in $\$ 5$ of the present paper will be concerned mainly with a number of preliminary experiments carried out to lay a proper foundation.

The second transition process, which will be called transition by spectral evolution, is typical of Couette flows dominated by rotation of the inner cylinder (the region above the origin of co-ordinates in figures $2(\mathrm{a}),(\mathrm{b})$ and (c)). As the speed increases, the flow as a whole undergoes a slow, superficially reversible passage through a limited sequence of regular but increasingly complicated patterns. The process begins with the Taylor instability. This is followed by a second instability which leads to the appearance of a tangential wave pattern rotating at approximately the mean speed of the two cylinders. The ensuing motion then remains laminar and doubly periodic for a time, as successively higher-order harmonics of the basic frequencies are excited. At sufficiently high speeds, however, the flow becomes noticeably no longer quite laminar, in the sense that irregularities have begun to appear, especially in the motions of smallest scale. Further increases in speed then increase the degree of irregularity until finally the flow can only be described as fully turbulent. In this same evolutionary process the spectrum is at first discrete, although it may become quite complex as new frequencies are added by non-linear interactions of existing modes. With increasing speed there is a gradual appearance of noise, which is to say a gradual broadening of the spectral lines, until the final spectrum is continuous, with perhaps some vague residual periodicities in the motions of largest scale. The essential feature of this process is the absence of intermittency, and the essential consequence is that no method is available for defining or measuring the degree of turbulence in the flow except in terms of the spectral broadening concept itself.

Finally, a different but equally important feature of transition by spectral 
evolution is an elegant and nearly unprecedented question of experimental uniqueness. Throughout the singly-and doubly-periodic régimes, it is found that a variety of different flows, characterized by different combinations of axial and tangential wave-number, can exist at a given speed. Which one of these will actually be observed in a particular experiment is determined by the whole operating history of the experiment, and especially by the rate and direction of approach to the speed in question. In these circumstances, there is little doubt that the issue of uniqueness hangs on the relative stability of alternative and closely equivalent modes, rather than on the absolute stability of a single mode. What is new in the present experiments is a sudden and inescapable emphasis on relative stability as a practical experimental matter, together with a set of observations which provide an accurate and reasonably complete description of the behaviour of the flow in one particular apparatus. The latter observations, which are set out at length in $\$ 4$, are viewed as the main contribution of this paper to the literature of hydrodynamic stability.

\section{Design and instrumentation of the large machine}

\subsection{Mechanical details}

The first apparatus constructed at GALCIT for the study of circular Couette flow was put into operation in 1956. The working fluid in this apparatus is air, and observations of the motion are made using standard techniques of hot-wire anemometry. The original objective, which was to make the machine as large as available funds would permit, was met and even slightly exceeded. The nominal diameters chosen for the inner and outer cylinders were 32 and $36 \mathrm{in}$., respectively; the nominal length was $60 \mathrm{in}$. The actual measured dimensions at $21^{\circ} \mathrm{C}$ are shown in figure 3 . The cylinders and shafts were mounted in a large gimbal frame to allow the axis of rotation to be changed from the normal horizontal position to a vertical one if necessary to cope with effects of free convection on hot-wire response at low speeds. One end of the gimbal frame could be opened for installation or removal of the cylinders, and a hoist was provided for handling the heavier components. The machine itself was supported from the ceiling in order to keep the floor area free for instrumentation and other uses.

With the object of increasing the rigidity of the cylinders and reducing their cost, each cylinder was assembled in five $1 \mathrm{ft}$. flanged sections. The material is a cast aluminium alloy chosen primarily for dimensional stability rather than for machinability, and a certain sacrifice in surface finish had to be accepted. The final finish shows circumferential grooves with an axial pitch of $0.010 \mathrm{in}$. and a depth of perhaps $0.0002 \mathrm{in}$., together with numerous minute air pockets resulting from the casting process. Inasmuch as the individual aluminium sections had to be machined while clamped by one flange to a steel machine bed, some distortion of the final surface resulted from unavoidable variations in temperature during machining. Before the cylinders were assembled, therefore, the diameter of each section was measured at various points around the circumference, and the two flanges to be joined were selected so that nearly equal and opposite deflexions would be required to force them into a true circular shape 
while the connecting bolts were installed. By this method nearly true cylinders were obtained at reasonable cost. The final runout of the cylinders is determined primarily by runout at the ends, where a set of adjustable spokes like those in a bicycle wheel is used to connect each cylinder to its shaft. The angle of these spokes was chosen so that negligible stresses would be produced by differential

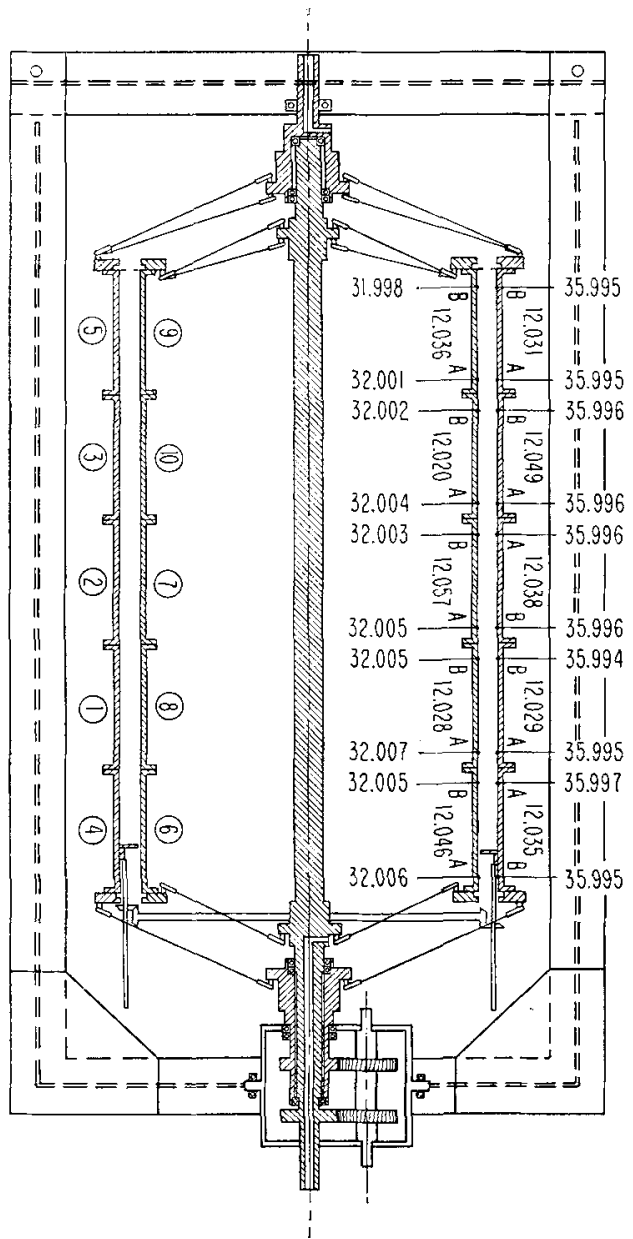

Figure 3. Plan view and section of large rotating-cylinder apparatus $\left(r_{o} / r_{i}=1 \cdot 125\right)$. Dimensions given are in inches at $21^{\circ} \mathrm{C}$.

thermal expansion of the aluminium and steel components. With reasonable care, the runout at any point on either cylinder can be made less than $\pm 0.00 \mathrm{I}$ to $\pm 0.002 \mathrm{in}$. This figure includes contributions from machining tolerances for shafts and cylinders, from bearing eccentricity, and from inaccuracies of adjustment.

\subsection{Problems in operation}

At the outset of the research, only three operating conditions were contemplated. These were (1) inner cylinder rotating, outer at rest; (2) outer cylinder rotating, inner at rest; (3) both cylinders rotating in the same direction, with irrotational 
flow in the working space. A gear box and a single driving motor were to be used for the three conditions mentioned. Because the interesting speeds for conditions (1) and (2) were very much different from each other, however, the original motor had to be replaced almost immediately by a larger motor and variable transmission to permit wider variations in speed. A second similar motor and transmission have since been added, and each motor is now coupled directly to the appropriate shaft, by-passing the gear box. A V-belt coupling was first tried for the sake of quietness, but unacceptable fluctuations in speed were observed. The belts were therefore replaced by timing chains, which are satisfactory as long as they are kept quite slack to minimize noise and vibration. The useful speed range for either cylinder lies between 1 r.p.m. and 400 r.p.m. This speed is monitored continuously with the aid of an electronic counter which measures the period between successive pulses generated once per revolution by a magnetic pickup. There is no provision for static or dynamic braking; in fact, over-running clutches are installed on the motor shafts to prevent power transmission in the reverse direction.

Once the cylinders had been equipped for independent operation, it was found that if only one cylinder rotated it could be made to run true, but if both cylinders rotated neither ran true, as if the central shaft were not straight. On the other hand, the various components of the bare shaft had run almost perfectly true before the installation of the cylinders. The trouble was finally traced to the fact that the spokes used to support the cylinders were attached alternately to opposite sides of the hub flange, as shown at the top in figure 3. Since the spokes were not all under the same tension, a substantial couple could be applied to the shaft at the hub-attachment point. The solution was to move all the spokes to the same side of the hub flange, as shown at the bottom in figure 3. Although the spokes now necessarily run in a radial direction in an end view, no difficulty arises in transmitting the required torques from the shafts to the cylinders. Quite recently, the deflexion of the central shaft under the weight of the two cylinders has been measured. According to these measurements, the axis of rotation of the inner cylinder lies $0.006 \mathrm{in}$. below the axis of the outer cylinder.

Finally, since the instrumentation is usually unsymmetrical, some means had to be found to balance the cylinders, especially for very high and very low speeds. This has been accomplished by using a switch, actuated by the teeth of the shaft gear, to detect variations in speed during a single revolution. By adding appropriate weights (the effect of a $10 \mathrm{~g}$ weight at the rim of the $70 \mathrm{~kg}$ cylinder is readily observable), these variations can be made less than 2 or $3 \%$ at a speed of 1 r.p.m., which is roughly the inner cylinder speed for Taylor instability.

\subsection{Instrumentation and probe-traversing mechanisms}

The means provided for making electrical connexions to the inner and outer cylinders, through the bottom and top ends of the shaft assembly, respectively, are shown in figure 3. At first, measurements were made with one stationary cylinder which also carried the hot-wire instrumentation. Later on, an 8-channel slip-ring assembly was added at each end of the main shaft. The electrical noise originating at these rings, although it varies with current and with speed, and 
also from one ring to another, is usually negligibly small compared to a typical hot-wire signal. At the present time, ten additional channels of slip-ring communication have been installed to accommodate more complex instrumentation.

Each of the ten cylinder sections is equipped with two removable pads $180^{\circ}$ apart for mounting probes. For various reasons it has sometimes been desirable to make radial traverses of the flow without stopping the machine. Such traverses are easily carried out if either cylinder is at rest. If both cylinders are rotating, however, a problem arises not in moving the probe but in keeping track of its radial position under adverse circumstances of high speed, large radial accelerations of $20 \mathrm{~g}$ or more, and occasional vibration due to unbalance of the heavy cylinders. This problem has been solved with the aid of remotely indicating traversing units driven by special motors which are supplied with $115 \mathrm{~V}$ $400 \mathrm{c} / \mathrm{s}$ power through the slip rings just mentioned. In each traversing unit the gear train driving the probe is arranged so that 10,000 motor revolutions correspond to $1 \mathrm{in}$. of radial travel. The special feature of these motors is the provision of an integral two-pole permanent-magnet generator producing an oscillating signal at the motor frequency of about $10,000 \mathrm{c} / \mathrm{min}$ or $160 \mathrm{c} / \mathrm{s}$. This signal is led out of the machine to an electronic counter which shows the probe radial position directly in inches. Inasmuch as the counter continues to add when the probe motion is reversed, a second counter is used to keep track of motion in the opposite direction. Two of the three motor-equipped instrumentation pads also have provision for adjusting the probe in pitch and yaw, in order to allow a complete hot-wire calibration in situ.

The last point of interest here concerns the occasional necessity for traversing hot-wire probes in the axial direction, again with the cylinders rotating. In early attempts to drag a light sled axially by means of cables extending through the end closures and serving also as electrical leads for a hot wire, it was found that the flow was greatly disturbed by the wake of the sled. Inasmuch as provision has already been made for mounting probes $1 \mathrm{ft}$. apart in the axial direction along both cylinders, it was decided to try to move the flow past these probes, rather than vice versa, by installing end closures which could themselves be traversed axially. The original sheet-metal end plates were split midway between the cylinders, as shown at the top in figure 3 , with half of each plate rotating with each cylinder. For mechanical reasons, and also to remove an undesirable vorticity concentration at the mid-radius on the end-walls (the kind of private instability which can occur in this vorticity layer is shown in several of the photographs in plates 1-12), the new end-plates span the working space and rotate with the outer cylinder. These new closures are transparent to allow probe radial positions to be checked optically with a cathetometer. Each end-plate can be independently moved in the axial direction by a set of four ball screws having rotating nuts connected by shafts as shown at the bottom in figure 3 . Power is supplied either directly, if the outer cylinder is at rest, or through slip rings, if the outer cylinder is rotating. The total travel of each end-plate is 14 in., and the position is indicated by an aluminium ring which also helps to support the free end of the ball screws. 


\section{Design and instrumentation of the small machine}

\subsection{Mechanical details}

The second apparatus for the study of circular Couette flow has glass cylinders and is roughly a $1 / 8$-scale model of the first one. The major difference is that the cylinders are supported at one end only, because of the need for access to the interior for optical instrumentation. The general arrangement of the apparatus

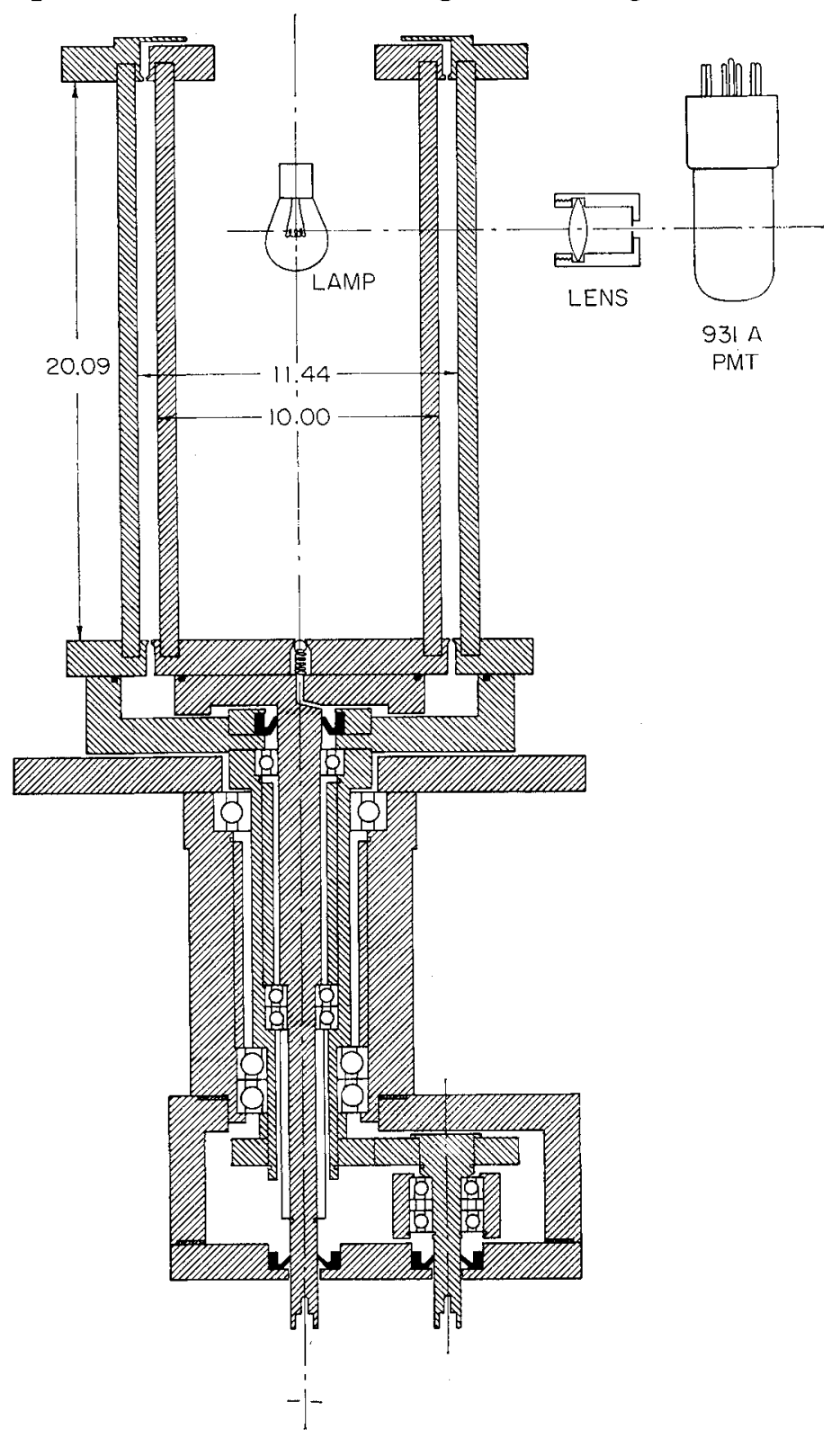

FIGURE 4. Section of small rotating-cylinder apparatus. Dimensions given are in $\mathrm{cm}$ for precision cylinders $\left(r_{o} / r_{i}=1 \cdot 144\right)$. Early experiments with this apparatus used rough cylinders $\left(r_{o} / r_{i}=1 \cdot 135\right)$. 
is shown in figure 4. This small machine was put into operation late in 1957. The electric motors used have good speed regulation in the range between 5 and $100 \%$ of full speed, and can be readily changed from one to another of three gear ratios to provide any speed from 3 to 1600 r.p.m. in either direction for either cylinder. This speed is measured by using a magnetic pickup, energized by a 60-tooth gear on the motor shaft, to drive an electronic counter.

\subsection{Flow-visualization methods and optical instrumentation}

The working fluid in the small machine is a silicone oil which is commercially available in various viscosity grades. The flow-visualization technique is the same one used by Schultz-Grunow \& Hein (1956), and earlier in a somewhat diluted form by Terada \& Hattori (1926), Lewis (1928), and Hagerty (1946). When a small amount of aluminium paint pigment is suspended in the oil, the minute flakes of metal take up, on the average, a preferred orientation which is almost certainly determined by the direction of the principal axes of the local rate-of-strain tensor. For a very slow laminar flow, for example, observations with an ordinary microscope suggest that the flakes are oriented at a constant angle with respect to the walls, except for an occasional rapid rotation by $180^{\circ}$ at what seem to be random intervals. In more complicated flows, variations in particle orientation affect the optical properties of the suspension for both reflected and transmitted light. The aluminium pigment used here consists of irregular flakes with a typical maximum dimension of $10^{-3}$ in. and a thickness (estimated from the manufacturer's figure for covering power on a water surface) of perhaps $10^{-5} \mathrm{in}$. The particles tend to settle out (half in about $2 \mathrm{~h}$ for a kinematic viscosity of $0.01 \mathrm{~cm}^{2} / \mathrm{sec}$; half in about $48 \mathrm{~h}$ for $0.20 \mathrm{~cm}^{2} / \mathrm{sec}$ ) when the fluid is at rest, but a brief period of operation with turbulent flow restores the uniformity of the suspension. Centrifuging of the pigment at high speeds for the less viscous fluids is a major factor in limiting the Reynolds numbers for which this flow-visualization technique is useful. It has also been found that the pigment may sometimes tend to adhere to the glass surfaces of the apparatus, unless scrupulous care is taken in removing all traces of oil, solvents, and other contaminants before assembly.

Previous applications of the suspended-particle method of flow visualization seem to have made use only of reflected light. In the present work, however, an unpremeditated but highly successful extension of the technique has been made by using transmitted light to obtain quantitative information about the flow structure. The optical arrangement is indicated in figure 4. Light from an incandescent lamp inside the cylinders passes through the fluid to a photomultiplier tube outside. The light beam is restricted to a diameter of about $0.5 \mathrm{~mm}$ by a lens which forms an image inside the fluid of a diaphragm actually located between the lens and phototube. With an optical path length in the fluid of about $7 \mathrm{~mm}$, a pigment concentration of about $0.0005 \mathrm{~g} /$ c.c. produces quite satisfactory signals. No effect of the pigment on the apparent viscosity could be detected in a laminar flow of the same fluid through a capillary-tube viscometer, even for appreciably heavier concentrations.

More recently this same optical technique has been adapted to another purpose. A piece of film is wrapped around the outer cylinder, and a line light source is 
placed along the axis. A baffle around the light source passes light only in a direction nearly normal to the axis of rotation. The original baffle, consisting of alternate layers of black paper and clear plastic, was completely ineffective because of total light reflexion from the plastic-paper interfaces. The assembly was transparent even when viewed at a quite oblique angle, and the paper might as well have been omitted. The plastic surfaces were therefore roughened by sandblasting before assembly, and the baffle is now satisfactory except that the thickness of the plastic spacers is not quite uniform. The lamp behaves everywhere along its length like a concentrated area source with a dimension of about $1.5 \mathrm{~mm}$. Little would be gained by attempting to reduce this dimension further, because the unavoidable separation of film and fluid, as well as the relative motion, prevent the resolution of details of the motion having a scale of less than about 0.01 in.

Two shadowgraphs obtained with this system are shown in figure 14 (plate 1). The film used was chosen primarily for its dimensional stability rather than for its speed or resolving power. The main object in taking these pictures was to verify that light intensity, baffle geometry, particle density in the fluid, film sensitivity, and development procedure were reasonably well matched. It is evident from the shadowgraphs that a photometer trace in the circumferential direction would be entirely equivalent to a direct light-probe signal. The shadowgraph, however, allows traces to be made in other directions as well. In fact, a useful application of the method may well be found in the measurement of axial and tangential correlation data by the optical method of Kovasznay (1949).

\subsection{Recent modifications}

The glass cylinders used in the initial experiments were made of commercial tubing and were far from round. The working space was $7.60 \mathrm{in}$. long, with inside and outside diameters of $3.998 \pm 0.005 \mathrm{in}$. and $4.535 \pm 0.015 \mathrm{in}$, respectively. Thus the nominal thickness of about $0.27 \mathrm{in}$. for the working space varied by several $\%$ from one point on the circumference to another, with a corresponding variation in the optical density of the fluid. Under these conditions, measurements with transmitted light were quite difficult because of refraction errors and variations in background signal. The flow itself was also affected by surface irregularities and by vibration at high speed. Consequently, the original cylinders have been replaced by a pair of precision ground and polished glass cylinders with a working space having the dimensions shown in figure 4 . The runout for either cylinder can be kept below a value of about $\pm 0.001 \mathrm{in}$. by care in assembly.

A further modification of the drive system for the small machine has also been made in an effort to obtain useful torque data. Each cylinder is now driven through a coupling consisting of two sections capable of rotating with respect to each other against a spring torque. Mirrors on the two sections alternately reflect a light beam onto a photocell, allowing the angular deflexion of the coupling to be measured during operation. This arrangement obviously yields only the total torque, including that required to overcome friction in the seals and bearings. 


\section{Transition by spectral evolution; the 'state' problem}

\subsection{The Taylor boundary}

As soon as the small Couette-flow apparatus was completed in late 1957, the flow-visualization technique described in $\$ 3$ was used to verify the position of the Taylor stability boundary. The result is shown by the open circles in figure 2 , in which the co-ordinates $R_{i}=\omega_{i} r_{i}^{2} / \nu$ and $R_{o}=\omega_{o} r_{o}^{2} / \nu$ are the usual Reynolds numbers based on radius and surface speed for the inner and outer cylinders respectively. Some of the scatter in these early observations is due to out-of-roundness of the cylinders or to poor control of temperature. Some uncertainty about the position of the boundary also arises from the fact that the cellular secondary motion does not appear simultaneously all along the length of the apparatus, but begins in the vorticity concentration at the ends and only reaches the centre after a slight increase in speed.

Three photographs taken with a spark-gap light source and reproduced in plate 2 show the secondary vortex pattern at the three points marked A, B, C respectively in figure $2(\mathrm{~b})$. In particular, the weak spiral configuration at the point $\mathrm{C}$ seems to be quite typical of Taylor instability for opposite rotation of the two cylinders in this apparatus, except at low Reynolds numbers for the outer cylinder. When the strength of the secondary motion is moderately strong with opposite rotation, one layer of cells can occasionally be distinguished spiralling up in the fluid while another layer is spiralling down.

The fourth photograph in plate 2 is a single frame from the motion picture prepared early in 1958. This photograph illustrates the secondary vortex structure at the point $D$ in figure 2 (c), well above the Taylor boundary, as viewed with transmitted light. $\uparrow$ The light source was a strong lamp placed inside the cylinders; the inner surface of the inner cylinder was covered with a thin sheet of translucent white plastic as a light diffuser. The feature of special interest in this photograph with transmitted light is the lens-shaped inclusion near the centre of the flow, where a single vortex filament doubles back on itself and closes at another point on the circumference-in this case, on the far side of the apparatus. Irregularities of this lens type sometimes occur during a rapid transit of the stability boundary (necessary here because of the limited period of operation of the motion-picture camera), but tend eventually to work themselves out, usually by diminishing in length and disappearing.

\subsection{The second boundary}

The position of a second stability boundary separating two distinct régimes of flow is shown by the filled circles in figure 2 . When this boundary is crossed (at least at any point to the right of the triple intersection at $R_{i}=2500, R_{o}=-4500$ in the left quadrant of figure $2(b)$ or (c)), the new motion which appears is a rotating pattern of tangential waves superimposed on the original cellular

$\dagger$ In interpreting this and other photographs of the Taylor motion, it need only be kept in mind that the aluminium flakes in the fluid are seen roughly face-on near the centre of the vortex cells and roughly edge-on near the boundaries between cells. The latter regions are therefore relatively transparent to transmitted light but appear dark when viewed by reflected light, with some differences also noticeable between stagnation and separation lines at the surface of the cylinders. 
motion. This is the doubly-periodic flow which was first described qualitatively by Taylor (1923) and later photographed by Schultz-Grunow \& Hein (1956). As demonstrated by the first of the two shadowgraphs in plate 1, an important feature of this doubly-periodic flow is a marked phase shift for alternate cell boundaries. Provided that the visible cell boundaries are stream surfaces (as
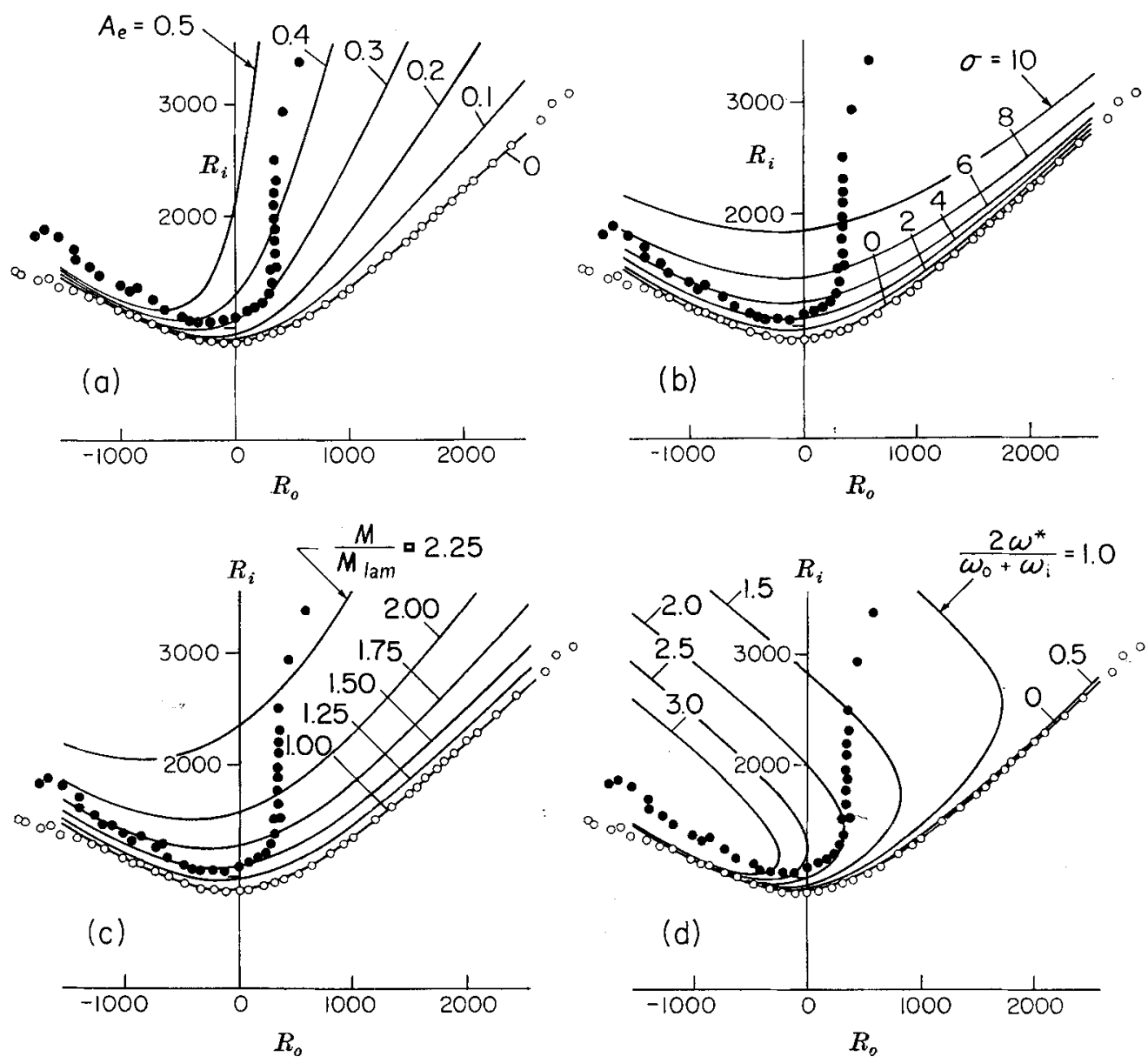

Figure 5. Computations according to Davey's analysis (1962). Co-ordinates and experimental data are as in figure 2 (c) (rough cylinders; $1.29<r_{o} / r_{i}<1.40$ ). Computations are for $r_{o} / r_{i}=1 \cdot 29$. Parameter in (a) is equilibrium amplitude $A_{e}$ from Davey's equation (7.1.11) with $m$ replaced by $m r_{o}^{2} / r_{i}^{2}$. Parameter in (b) is amplification factor $\sigma$ from Davey's equation (7.1.7). Parameter in (c) is ratio of torque to laminar torque (cf. figure 1). Parameter in (d) is ratio of characteristic frequency for secondary rotation, $\omega^{*}$, to mean frequency for basic flow, $\frac{1}{2}\left(\omega_{0}+\omega_{i}\right)$.

they are in the singly-periodic Taylor motion), and provided that the mean tangential flow does not vanish in a co-ordinate system rotating with the wave pattern, the new secondary flow is essentially a combined rotation and translation in a stream tube of periodically varying area.

Except for one paper by DiPrima (1961), no attempt has been made so far to treat this second instability theoretically. Among the major obstacles to progress 
is uncertainty about the actual flow at the onset of the instability, in spite of some important explorations of the non-linear Taylor régime which have been carried out by Stuart (1958) and Davey (1962) from a base on the Taylor boundary. Their method is to represent the secondary motion by the eigenfunctions of linearized theory (including certain essential second-order terms in the case of Davey's paper) and to determine the amplitude by an energy balance. For the present geometry, Davey's analysis implies the equilibrium amplitude $A_{e}=v^{*} / \omega_{i} r_{i}$ shown in figure 5(a), where $v^{*}$ is the modulus of the first-order perturbation in tangential velocity. Figure $5(\mathrm{~b})$ shows the corresponding linear amplification factor $\sigma$, defined in terms of an initial disturbance growth like $\exp \left(\sigma \nu t / d^{2}\right)$, where $d$ is the gap; and figure 5 (c) shows the relative torque $M / M_{\text {lam }}$ (cf. also figure 1).

A vague clue to the nature of the tangential instability is provided by the fact that wave motions are known to occur in a confined fluid undergoing combined translation and rotation if the helix angle of the bounding streamlines is steep enough (Fraenkel 1956). Another and probably related criterion permits wave motions in rotating fluids when the frequency of rotation is large enough compared to some disturbing frequency (Görtler 1944). To test the usefulness of such criteria in the present problem, the quantity $\omega^{*}=|\partial u / \partial z|_{\max }$ has been taken as a measure of the intensity of rotation about a tangent within the Taylor cells, and curves of constant frequency ratio $2 \omega^{*} /\left(\omega_{o}+\omega_{i}\right)$ have been computed and plotted in figure $5(\mathrm{~d}) . \dagger$ Whatever they may be worth as an attempted fit to the second stability boundary in figure $5(\mathrm{~d})$, these curves are computed for fixed geometry and are therefore also curves of constant helix angle $\omega^{*} d /\left(\omega_{o}+\omega_{i}\right) r_{i}$ (say). The idea of a critical helix angle, however, is not consistent with an impression produced by the available experimental data, that the two stability boundaries tend to approach each other $\ddagger$ as $r_{o} / r_{i} \rightarrow 1$.

$\dagger$ The shape of these curves depends on the fact that the theory makes the angular velocity $\omega^{*}$ increase at first more rapidly than the mean cylinder velocity but then become asymptotically constant. The latter behaviour is almost certainly not realistic, inasmuch as the theory also requires the amplification factor $\sigma$ to be small compared (probably) to $\pi^{2}$, and according to figure 5 (b) such a condition is only satisfied quite near the Taylor boundary. For other reasons, the theory also becomes less accurate when moving from right to left in the figure, and fails altogether for strong opposite rotation of the two cylinders in the left quadrant. Hence the region in which Stuart's or Davey's analysis is capable of describing the real motion is quite limited, and includes at most only a small fragment of the second boundary where the speed of the outer cylinder is relatively low,

$\$$ The present speculations, which are in no sense an explanation of the tangential instability, can be criticized on numerous grounds. Nevertheless, it is worth noting that Davey's analysis for the limiting case of a small gap/radius ratio with $\omega_{0}=0$ can be used to justify a formula

$$
\left(\frac{2 \omega^{*}}{\omega_{i}}\right)_{c}=1 \cdot 86\left[\frac{r_{i}}{d}\left(1-\frac{T_{c}}{T_{c}^{\prime}}\right)\right]^{\frac{1}{2}},
$$

where $T_{c}$ and $T_{c}^{\prime}$ are Taylor numbers for the first and second instabilities, respectively. Some Chicago data (Donnelly 1963) give the experimental value $T_{\mathrm{o}}^{\prime} / T_{\mathrm{c}}=\left(\omega_{c}^{\prime} / \omega_{c}\right)^{2}=1 \cdot 15$ for $r_{o} / r_{i}=1 \cdot 053$, and the present GALCIT data for precision cylinders give $T_{c}^{\prime} / T_{c}=1.55$ for $r_{o} / r_{i}=1 \cdot 144$. In both cases the formula just cited yields a value $\left(2 \omega^{*} / \omega_{i}\right)_{c}=2 \cdot 9$. These results provide a tentative empirical criterion for the occurrence of the second instability, including, incidentally, a prediction that the conditions for instability cannot be satisfied if $r_{o} / r_{i}$ is greater than about $\mathbf{1 \cdot 4}$. 
Further details of some typical doubly-periodic motions are illustrated in plates 3 and 4. The first two photographs in plate 3 , taken from the motion picture mentioned earlier, are front-lighted and back-lighted views of the flow at the point $\mathrm{E}$ in figure $2(\mathrm{c})$. It should be noted that similar constraints are applied to the motion at the two ends, in spite of the fact that the fluid is bounded below by solid walls and above by a free surface. Closeup views of this same motion are reproduced in plate 4.

The third photograph in plate 3, taken with the spark-gap light source, shows a complex (but completely correlated, and hence technically laminar) doublyperiodic flow at the point $F$ in figures $2(a)$ and (b), well above the first and second stability boundaries. The fourth photograph in plate 3 shows a nominally singlyperiodic flow at the point $G$ in figure $2(\mathrm{~b})$. In this case, however, it appears that a higher-order tangential mode has been excited before the fundamental. + This behaviour seems to be typical of the more energetic flows close to the doublyperiodic boundary for cylinders rotating in the same direction, and the nature of the second or tangential instability in this region is therefore not yet clear.

\subsection{The tangential wave velocity; uniqueness}

It is an experimental fact in figure 2 (c) that the boundary separating the singlyand doubly-periodic flow régimes in the right quadrant is not unique, but depends on an axial wave-number which can itself take on several discrete values. This property of non-uniqueness for the circular Couette flow was noticed quite early in the present research, to be sure, but its importance was at first completely overlooked. When precision glass cylinders finally became available in early 1960 , a closer study began of the doubly-periodic motions for the case of the inner cylinder rotating and the outer cylinder at rest. Particular attention was paid at first to the characteristic angular velocity of the tangential waves. The results, shown in figure 6 , include data obtained in the small apparatus using a light probe and photomultiplier tube to measure phase velocity and using photographic or other means to determine wave number. Also shown are data obtained by C. Van Atta in the large apparatus using hot-wire anemometers to determine the same quantities through observations of the flow from both cylinders. $\ddagger$ When the waves first appear, their angular velocity is close to half the angular velocity of the rotating inner cylinder, in agreement with a tentative theoretical

$\dagger$ At sufficiently high speeds, a private instability leading to tangentially periodic disturbances can occur in the vorticity anomaly at the ends of the working space. In several of the spark photographs, which were taken with the space between the cylinders completely filled with fluid, these disturbances can be seen at both top and bottom of the apparatus.

\$ The relatively large scatter in these measurements in air is almost unavoidable. Toward the low-speed end of the experimental range in figure 6 (b), the inner cylinder is rotating at perhaps 6 r.p.m., and the wave pattern is rotating at about one-third of this speed. The wave periods which have to be measured are therefore of the order of several seconds. Under these conditions, moreover, the relative velocity at the hot-wire probes is less than $10 \mathrm{~cm} / \mathrm{sec}$, and the heat loss from the wires is dominated by free rather than forced convection. If the probe happens to be on the horizontal inner cylinder, for example, large but irrelevant periodic signals are generated solely by the steady rotation of the velocity vector with respect to the direction of the gravitational field. 
estimate by DiPrima (1961). As the speed increases, however, the dimensionless wave velocity rapidly approaches a value of approximately 0.34 in both machines. This latter value is then practically constant (i.e. independent of Reynolds number) out to the present limit of observation, where the noise level

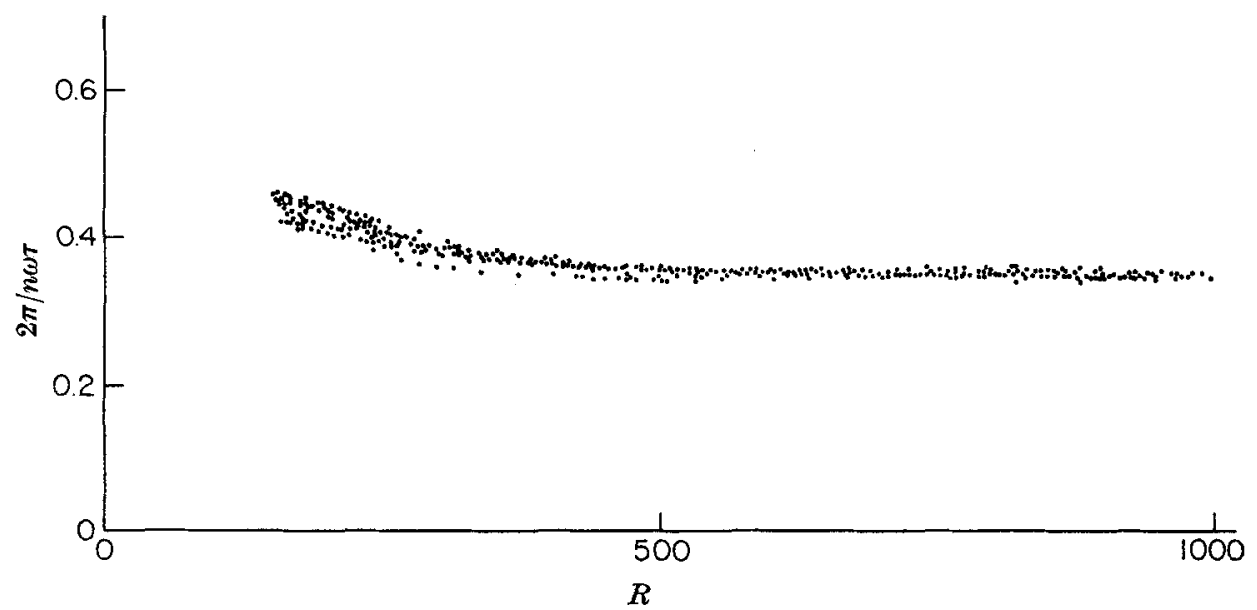

(a)

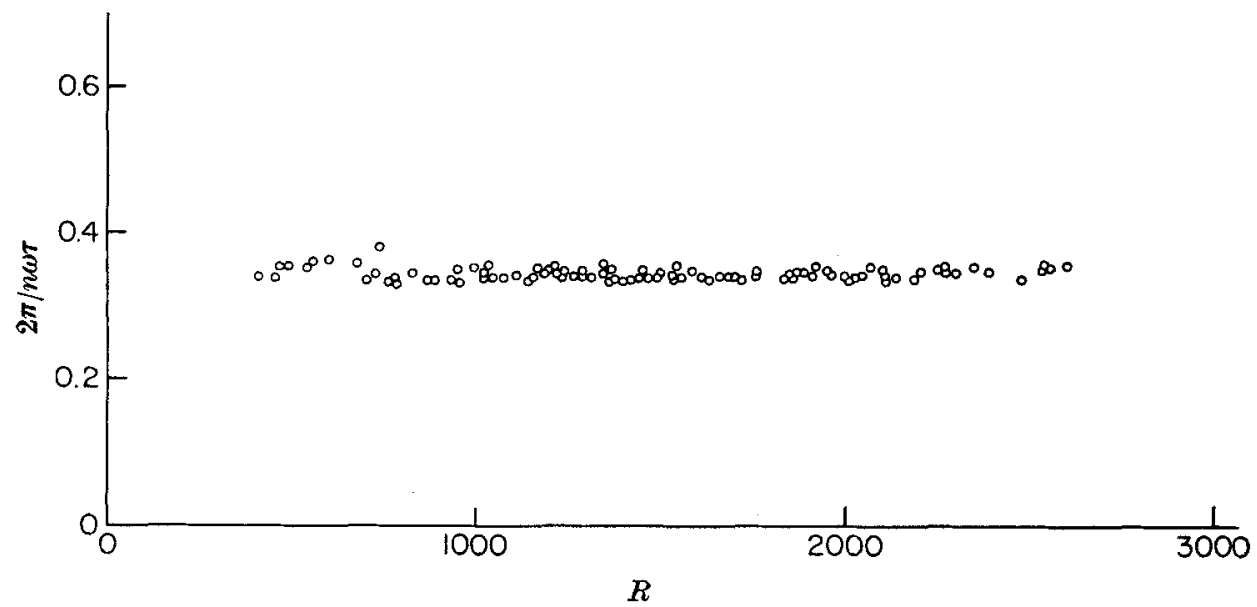

(b)

Figure 6. Relative angular velocity of the tangential wave pattern in doubly-periodic Couette flow when the outer cylinder is at rest. Reynolds number $R=\omega_{i} r_{i}\left(r_{0}-r_{i}\right) / \nu$ is based on gap and on surface speed of inner cylinder. Date in (a) are for silicone oil in small apparatus (precision cylinders; $\left.r_{o} / r_{i}=1 \cdot 144\right)$. Data in (b) are for air in large apparatus $\left(r_{o} / r_{i}=1 \cdot 125\right.$ ). In both cases the number of tangential waves may be $3,4,5,6$, or 7 in various ranges of $R$.

is so high that a dominant frequency for the largest-scale motions can no longer be isolated by conventional filtering techniques.

In the course of these measurements of wave velocity, a fascinating peculiarity of the Couette flow was discovered. As the speed of the inner cylinder was slowly increased or decreased in the doubly-periodic régime, with the outer cylinder at 
rest, the flow pattern was observed to change abruptly, discontinuously, and irreversibly from one state to another at certain well defined and repeatable critical speeds. The term 'state' is used here in a deliberately vague sense, the state variables being taken at least temporarily as the two integers denoting the number of Taylor cells and the number of tangential waves respectively. A change of state, or a transition, then implies a discrete change in one or both of these integers. The actual physical processes by which the number of cells or the number of waves increases or decreases will be described in detail in $\$ 4.5$.

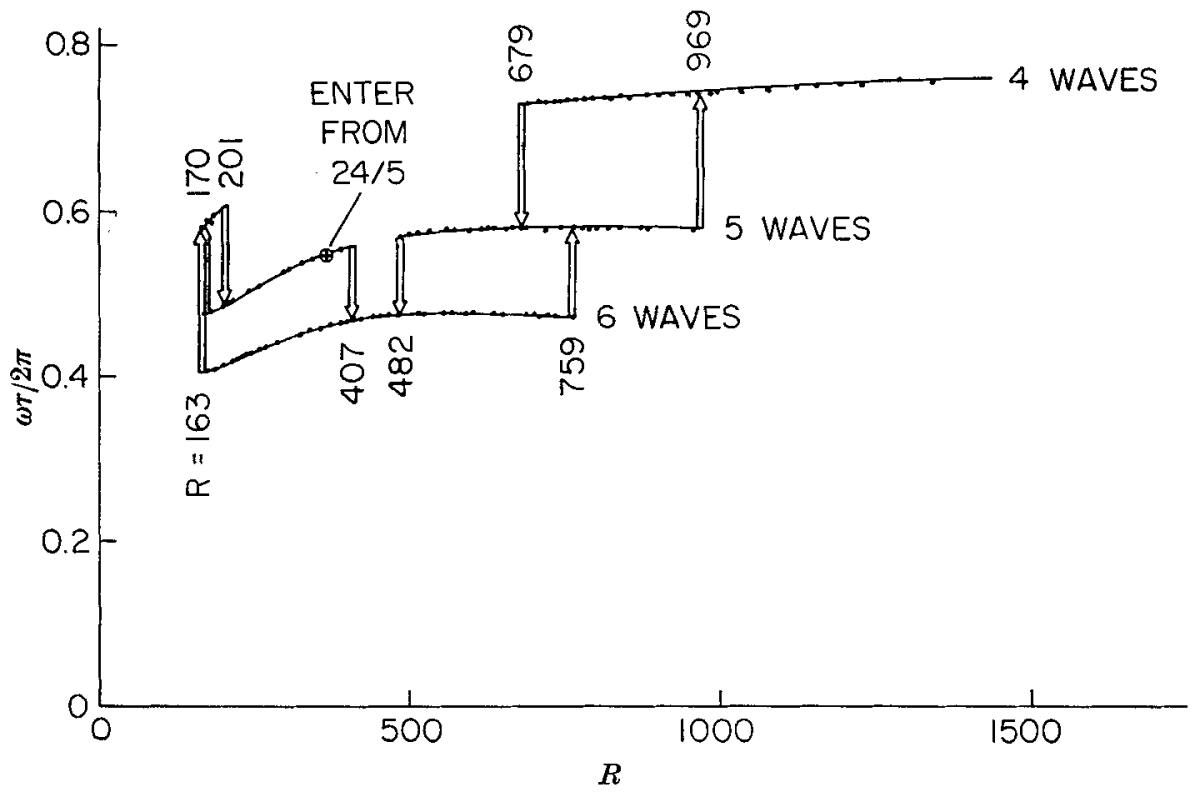

FIGURE 7. Hysteresis loops in state diagram for 22 Taylor cells (precision cylinders; $\left.r_{o} / r_{i}=1 \cdot 144\right)$. Speed of inner cylinder is varying slowly with outer cylinder at rest. Ordinate is ratio of period of wave passage $\tau$ to period of cylinder rotation $2 \pi / \omega$. Abscissa $R$ is Reynolds number based on gap and on surface speed of inner cylinder.

The property of non-uniqueness is most vividly demonstrated by the existence of a number of hysteresis loops, in which the flow changes from one state to another and back again as the speed of the inner cylinder is slowly increased and decreased. $\dagger$ Several such hysteresis loops, involving changes in tangential wavenumber only, are shown in figure 7 . The ordinate in this figure is the ratio of the period of the tangential wave passage, $\tau$, to the period of rotation of the inner cylinder, $2 \pi / \omega$, both measured in laboratory co-ordinates. These are raw data,

$\dagger$ Other physical situations are of course known in which rotational fluid motions show a tendency toward hysteresis or non-uniqueness. Examples which come readily to mind include: the stalling behaviour of many airfoil sections; Pai's (1943) study of turbulent Taylor cells in Couette flow; Roshko's (1955) study of boundary-layer flow past a cavity of variable depth; Fultz's (1961) study of tangential wave patterns in a rotating annulus in the presence of radial heat transfer. Wave patterns occurring in cellular convection between horizontal surfaces must also exhibit this same kind of behaviour. In none of these cases, however, is the property of non-uniqueness so conspicuous, or so well documented, or so compelling of attention, as in the experiments reported here. 
incidentally, of the kind that first called attention to the existence of state transitions involving the tangential wave number. No attempt has been made so far to modify or control the form of these hysteresis loops, e.g. by changing the noise level in the environment.

In view of the tangible non-uniqueness of Couette flow, the measurements of tangential wave velocity in figure 6 are remarkable for the absence of dispersion. The normalized wave velocity is so nearly independent of speed, and at fixed speed is so nearly independent of wave number, that it is essentially an invariant of the whole experiment. These measurements extend well into the transition régime, where a dominant tangential wave-number is most easily detected by observing the heavily damped motion very near the ends of the apparatus (note the position of the lamp, lens, and phototube in figure $19(\mathrm{f})$, plate 7$)$. The results therefore suggest that doubly-periodic Couette flow may provide a model for study of at least one important property of turbulent flow; namely, the characteristic velocity of the large-eddy structure. $\dagger$ At the same time, the present data are a warning that an eddy which is continuously exchanging fluid with its environment, meanwhile operating on the vorticity of this fluid so as to preserve the local flow pattern, cannot usefully be viewed either as a quasi-solid-body motion or as a quasi-two-dimensional motion in which vorticity remains permanently attached to each fluid element (except for the diffusive effects of viscosity) during distortion.

\subsection{The state problem}

For obvious reasons, as soon as the nature of the problem had been realized, the investigation of state transitions became for a time the central object of the experimental work. For the particular apparatus used in these experiments, and for the range of speeds up to about 14 times the critical speed for Taylor instability, a total of 74 such transitions were definitely identified. Each was observed 3 or more times, the critical Reynolds number being repeatable in most cases within 2 or $3 \%$. The same fluid, a silicone oil with a kinematic viscosity of $0.213 \mathrm{~cm}^{2} / \mathrm{sec}$ at $21^{\circ} \mathrm{C}$, was used throughout.

In figure 8 , which is in the nature of a generalization of figure 7 to three dimensions, an attempt has been made to show entire this complex phenomenon of state transitions. The base of figure 8 is a rectangular grid laid off in one direction according to the number of Taylor cells in the flow, and in the other direction according to the number of tangential waves. The height above the base is taken proportional to the speed of the inner cylinder. Each transition can then be represented by a horizontal bridge or arrow from one column to another, indicating the nature and direction of the transition in wave-number space as well as the critical speed. Information equivalent to this figure is also presented in table 1.

$\dagger$ It is obviously desirable to extend these measurements of wave velocity to flows with both cylinders rotating, including certain fully turbulent flows which are known to show a dominant tangential as well as axial wave number. An example is provided by the motion at the point $I$ in figure 2 (a) (plate 5(i)). A slowly rotating tangential wave pattern in this flow is clearly visible in the motion picture mentioned earlier. 
Inner cylinder rotating; outer cylinder at rest.

Gap/inner radius $=0.144 ;$ length/gap $=27.9$

\section{Reynolds number}

\begin{tabular}{|c|c|c|c|c|c|}
\hline$\underset{\begin{array}{c}\text { Increasing } \\
\text { speed }\end{array}}{R=\omega_{i} r_{i}}$ & $\underbrace{\left(r_{o}-r_{i}\right) / \nu}_{\begin{array}{c}\text { Decreasing } \\
\text { speed }\end{array}}$ & $\begin{array}{c}\text { Initial } \\
\text { state } \\
\text { (cells/waves) }\end{array}$ & $\begin{array}{c}\text { Final } \\
\text { state } \\
\text { (cells/waves) }\end{array}$ & $\begin{array}{l}\text { Number } \\
\text { of } \\
\text { accessible } \\
\text { states }\end{array}$ & $\begin{array}{c}\text { Expected } \\
\text { state } \\
\text { (cells/waves) }\end{array}$ \\
\hline 114 & 一 & $0 / 0$ & $28 / 0$ & & Taylor boundary \\
\hline 143 & 一 & $28 / 0$ & $28 / 4$ & $10 t$ & Wave boundary \\
\hline - & 144 & $22 / 4$ & $26 / 4$ & 11 & $27 \cdot 5 / 4 \cdot 5$ \\
\hline- & 152 & $26 / 6$ & $26 / 4$ & & \\
\hline- & 152 & $30 / 6$ & $30 / 4$ & 13 & $27 \cdot 5 / 4 \cdot 7$ \\
\hline 一 & 154 & $28 / 6$ & $28 / 4$ & & \\
\hline- & 154 & $32 / 6$ & $32 / 4$ & 15 & $27 \cdot 9 / 4 \cdot 9$ \\
\hline- & 158 & $24 / 6$ & $24 / 4$ & 16 & $27 \cdot 6 / 4 \cdot 9$ \\
\hline- & 163 & $22 / 6$ & $22 / 4$ & & \\
\hline- & 163 & $26 / 7$ & $26 / 5$ & 18 & $27 \cdot 2 / 5 \cdot 1$ \\
\hline- & 164 & $28 / 7$ & $28 / 5$ & 19 & $27 \cdot 3 / 5 \cdot 2$ \\
\hline- & 165 & $30 / 7$ & $30 / 5$ & 20 & $27 \cdot 4 / 5 \cdot 3$ \\
\hline- & 167 & $24 / 7$ & $24 / 5$ & 21 & $27 \cdot 2 / 5 \cdot 4$ \\
\hline- & 168 & $32 / 7$ & $32 / 5$ & 22 & $27 \cdot 5 / 5 \cdot 5$ \\
\hline$\longrightarrow$ & 170 & $22 / 5$ & $22 / 4$ & 23 & $27 \cdot 2 / 5 \cdot 4$ \\
\hline - & 177 & $22 / 7$ & $22 / 5$ & 24 & $27 \cdot 0 / 5 \cdot 5$ \\
\hline - & 188 & $20 / 5$ & $24 / 5$ & $\begin{array}{l}24 \\
25\end{array}$ & $26 \cdot 7 / 5 \cdot 5$ \\
\hline- & 200 & $20 / 6$ & $24 / 6$ & 26 & $26 \cdot 5 / 5 \cdot 5$ \\
\hline 201 & - & $22 / 4$ & $22 / 5$ & $\begin{array}{l}20 \\
25\end{array}$ & $\begin{array}{l}26 \cdot 5 / 5 \cdot 5 \\
26 \cdot 6 / 5 \cdot 6\end{array}$ \\
\hline 203 & - & $32 / 5$ & $30 / 5$ & 24 & $\begin{array}{l}26 \cdot 6 / 5 \cdot 6 \\
26 \cdot 4 / 5 \cdot 6\end{array}$ \\
\hline - & 206 & $20 / 7$ & $24 / 7$ & & \\
\hline- & 207 & $18 / 5$ & $22 / 5$ & $\begin{array}{l}25 \\
26\end{array}$ & $26 \cdot 2 / 5 \cdot 6$ \\
\hline 211 & - & $32 / 6$ & $28 / 6$ & 26 & $25 \cdot 9 / 5 \cdot 6$ \\
\hline 215 & - & $24 / 4$ & $24 / 5$ & 25 & $25 \cdot 6 / 5 \cdot 6$ \\
\hline 217 & - & $30 / 5$ & $28 / 5$ & 24 & $25 \cdot 7 / 5 \cdot 7$ \\
\hline 217 & - & $32 / 4$ & $28 / 4$ & & \\
\hline 219 & - & $30 / 6$ & $28 / 6$ & 22 & $25 \cdot 2 / 5 \cdot 8$ \\
\hline 225 & - & $32 / 7$ & $30 / 7$ & 21 & $25 \cdot 0 / 5 \cdot 8$ \\
\hline 226 & - & $30 / 4$ & $26 / 4$ & 20 & $24 \cdot 6 / 5 \cdot 7$ \\
\hline - & 228 & $18 / 6$ & $22 / 6$ & 19 & $24 \cdot 3 / 5 \cdot 8$ \\
\hline 239 & - & $28 / 6$ & $26 / 6$ & 20 & $24 \cdot 0 / 5 \cdot 8$ \\
\hline 244 & 一 & $28 / 5$ & $26 / 5$ & 19 & $23 \cdot 8 / 5 \cdot 8$ \\
\hline 244 & - & $30 / 7$ & $28 / 7$ & & \\
\hline 247 & 一 & $28 / 4$ & $24 / 5$ & 17 & $23 \cdot 2 / 5 \cdot 8$ \\
\hline 260 & - & $26 / 4$ & $26 / 5$ & 16 & $22 \cdot 9 / 5 \cdot 9$ \\
\hline 286 & - & $26 / 6$ & $24 / 6$ & 15 & $22 \cdot 7 / 6 \cdot 0$ \\
\hline 297 & - & $26 / 5$ & $22 / 5$ & 14 & $22 \cdot 4 / 6 \cdot 0$ \\
\hline 297 & - & $28 / 7$ & $26 / 7$ & & \\
\hline- & 334 & $28 / 7$ & $26 / 7$ & 12 & $21 \cdot 7 / 6 \cdot 0$ \\
\hline 366 & - & $24 / 5$ & $22 / 5$ & 13 & $22 \cdot 2 / 6 \cdot 1$ \\
\hline 407 & - & $22 / 5$ & $22 / 6$ & 12 & $22 \cdot 0 / 6 \cdot 2$ \\
\hline- & 453 & $26 / 6$ & $24 / 6$ & 11 & $22 \cdot 0 / 6 \cdot 3$ \\
\hline
\end{tabular}

+ For the ten states 24/4, 24/5, 26/4, 26/5, 28/4, 28/5, 30/4, 30/5, 32/4, 32/5, the tangential waves disappear as $R$ decreases from 144 to 143 ; the Taylor cells then disappear at $R \sim 114$. Finer details in this range have not been resolved.

TABLE 1. State transitions in doubly-periodic flow 
Inner cylinder rotating; outer cylinder at rest.

Gap/inner radius $=0 \cdot 144$; length/gap $=27 \cdot 9$

Reynolds number

\begin{tabular}{|c|c|c|c|c|c|}
\hline \multicolumn{2}{|c|}{$R=\omega_{i} r_{i}\left(r_{o}-r_{i}\right) / \nu$} & \multirow{2}{*}{$\begin{array}{c}\text { Initial } \\
\text { state } \\
\text { (cells/waves) }\end{array}$} & \multirow{2}{*}{\multicolumn{2}{|c|}{$\begin{array}{cc}\text { Final } & \begin{array}{c}\text { Number } \\
\text { of } \\
\text { state }\end{array} \\
\text { accessible } \\
\text { (cells/waves) } & \text { states }\end{array}$}} & \multirow{2}{*}{$\begin{array}{l}\text { Expected } \\
\text { state } \\
\text { (cells/waves) }\end{array}$} \\
\hline $\begin{array}{l}\text { Increasing } \\
\text { speed }\end{array}$ & $\begin{array}{l}\text { Decreasing } \\
\text { speed }\end{array}$ & & & & \\
\hline- & 453 & $30 / 7$ & $28 / 7$ & 13 & $22 \cdot 9 / 6 \cdot 3$ \\
\hline - & 464 & $18 / 4$ & $18 / 5$ & 14 & $22 \cdot 6 / 6 \cdot 1$ \\
\hline 一 & 482 & $22 / 5$ & $22 / 6$ & 15 & $22 \cdot 5 / 6 \cdot 1$ \\
\hline - & 515 & $28 / 6$ & $26 / 6$ & 16 & $22 \cdot 9 / 6 \cdot 1$ \\
\hline - & 534 & $24 / 5$ & $24 / 6$ & 17 & $22 \cdot 9 / 6 \cdot 0$ \\
\hline - & 562 & $20 / 4$ & $20 / 5$ & 18 & $22 \cdot 8 / 5 \cdot 9$ \\
\hline - & 590 & $26 / 5$ & $24 / 5$ & 19 & $23 \cdot 0 / 5 \cdot 8$ \\
\hline - & 624 & $30 / 6$ & $28 / 6$ & 20 & $23 \cdot 3 / 5 \cdot 9$ \\
\hline - & 650 & $28 / 5$ & $26 / 5$ & 21 & $23 \cdot 5 / 5 \cdot 8$ \\
\hline 667 & - & $24 / 7$ & $24 / 6$ & 20 & $23 \cdot 5 / 5 \cdot 8$ \\
\hline 671 & 一 & $22 / 7$ & $22 / 5$ & 19 & $23 \cdot 6 / 5 \cdot 7$ \\
\hline - & 679 & $22 / 4$ & $22 / 5$ & 20 & $23 \cdot 5 / 5 \cdot 6$ \\
\hline 725 & - & $26 / 7$ & $26 / 6$ & 19 & $23 \cdot 4 / 5 \cdot 5$ \\
\hline 732 & - & $20 / 7$ & $20 / 5$ & 18 & $23 \cdot 6 / 5 \cdot 4$ \\
\hline - & $756 \ddagger$ & $32 / 7$ & $28 / 6$ & 19 & $24 \cdot 0 / 5 \cdot 5$ \\
\hline 759 & - & $22 / 6$ & $22 / 5$ & 18 & $24 \cdot 1 / 5 \cdot 5$ \\
\hline- & 772 & $24 / 4$ & $22 / 4$ & 19 & $24 \cdot 1 / 5 \cdot 4$ \\
\hline 810 & - & $20 / 6$ & $20 / 4$ & 18 & $24 \cdot 3 / 5 \cdot 4$ \\
\hline$\overline{822}$ & 817 & $18 / 3$ & $18 / 4$ & 19 & $24 \cdot 0 / 5 \cdot 3$ \\
\hline 833 & $\overline{836}$ & $24 / 6$ & $24 / 5$ & 18 & $24 \cdot 0 / 5 \cdot 2$ \\
\hline$\overline{851}$ & $\begin{array}{l}836 \\
-\end{array}$ & $26 / 4$ & $22 / 4$ & 19 & $24 \cdot 1 / 5 \cdot 2$ \\
\hline 851 & - & $28 / 7$ & $28 / 6$ & 18 & $23 \cdot 9 / 5 \cdot 1$ \\
\hline $\begin{array}{l}866 \\
969\end{array}$ & - & $20 / 5$ & $20 / 4$ & 17 & $24 \cdot 1 / 5 \cdot 1$ \\
\hline $\begin{array}{l}969 \\
988\end{array}$ & - & $22 / 5$ & $22 / 4$ & 16 & $24 \cdot 3 / 5 \cdot 1$ \\
\hline $\begin{array}{l}988 \\
995\end{array}$ & - & $26 / 6$ & $\begin{array}{l}26 / 5 \\
18 / 3\end{array}$ & 15 & $24 \cdot 1 / 5 \cdot 0$ \\
\hline $\begin{array}{r}995 \\
1046\end{array}$ & - & $\begin{array}{l}18 / 5 \\
18 / 6\end{array}$ & $\begin{array}{l}18 / 3 \\
18 / 3\end{array}$ & 14 & $24 \cdot 6 / 5 \cdot 0$ \\
\hline $\begin{array}{l}1046 \\
1069\end{array}$ & - & $\begin{array}{l}18 / 6 \\
30 / 7\end{array}$ & $18 / 3$ & 13 & $25 \cdot 1 / 4 \cdot 9$ \\
\hline $\begin{array}{l}1069 \\
1091\end{array}$ & - & $\begin{array}{l}30 / 7 \\
28 / 6\end{array}$ & $30 / 6$ & 12 & $24 \cdot 7 / 4 \cdot 8$ \\
\hline 1091 & 二 & $\begin{array}{l}28 / 6 \\
32 / 7\end{array}$ & $28 / 5$ & 11 & $24 \cdot 4 / 4 \cdot 6$ \\
\hline $1119 \ddagger$ & - & $\begin{array}{l}32 / 7 \\
30 / 6\end{array}$ & $26 / 4$ & 10 & $23 \cdot 6 / 4 \cdot 4$ \\
\hline 1207 & - & $\begin{array}{l}30 / 6 \\
18 / 4\end{array}$ & $28 / 5$ & 9 & $22 \cdot 9 / 4 \cdot 2$ \\
\hline 1231 & 二 & $18 / 4$ & $18 / 3$ & 8 & $23 \cdot 5 / 4 \cdot 3$ \\
\hline 1348 & $\overline{1609}$ & $20 / 4$ & $\begin{array}{l}18 / 3 \\
18 / 3\end{array}$ & 7 & $24 \cdot 0 / 4 \cdot 3$ \\
\hline- & 1609 & $20 / 3$ & $18 / 3$ & $8 \S$ & $23 \cdot 5 / 4 \cdot 1$ \\
\hline
\end{tabular}

$\ddagger$ These two transitions are quite violent, and sometimes lead to a final state other than the one given.

$\S$ The eight accessible states for $R>1609$ are 18/3, 20/3, 22/4, 24/4, 24/5, 26/4, 26/5, and 28/5. All are quite noisy, and all persist to somewhat higher speeds without further transition.

TABLE 1. (continued)

It should be emphasized that the data of figure 8 and table 1 are limited to those state transitions which occur as the speed of the inner cylinder varies slowly. The initial state for each transition is considered as given, although in practice some of these initial states can themselves be realized only after abrupt changes in speed for the inner cylinder or after preliminary operation of the outer cylinder 
in one direction or the other. $f$ In any case, no effort has been spared to establish, by whatever means, as many as possible of the states which can maintain themselves indefinitely in the present apparatus for steady rotation of the inner cylinder alone. In the later stages of the investigation, this effort was guided by

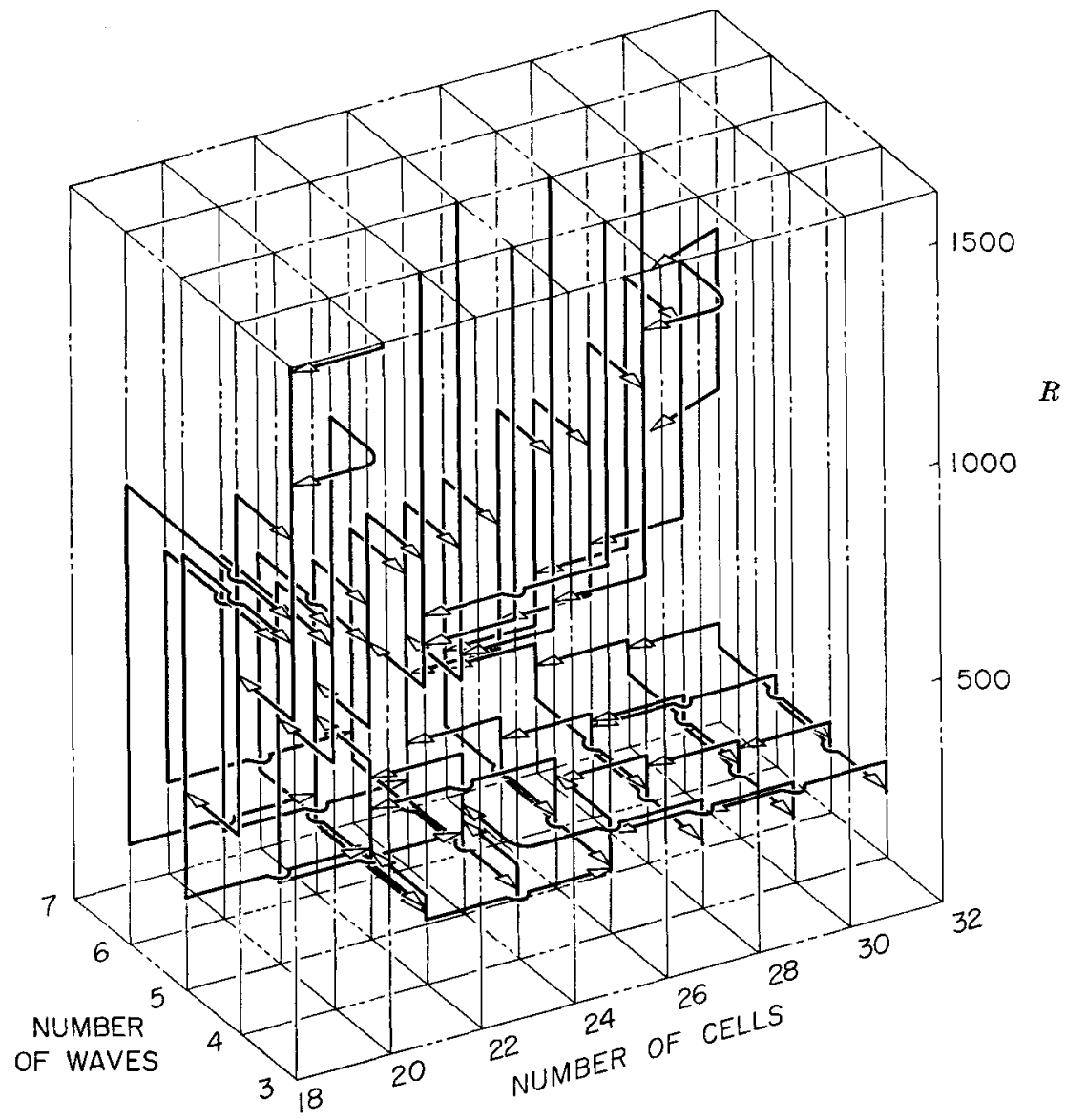

Figure 8. State transitions in doubly periodic Couette flow with outer cylinder at rest (precision cylinders; $r_{o} / r_{i}=1 \cdot 144$ ). Ordinate $R$ is Reynolds number based on gap and on surface speed of inner cylinder. Geometry and fluid properties are fixed. For tabulation of these data, including Reynolds number for each transition, see table 1.

$\dagger$ It is worth noting that the hysteresis loops in figure 7 are accessible with only very slow changes in speed starting from rest. As the speed of the inner cylinder is slowly increased from zero, 28 Taylor cells appear at $R=114$, followed by four waves at $R=143$. At $R=247$, four of the cells drop out in two separate pairs, and the number of waves simultaneously changes to five (this transition makes a knight's move in figure 8). At $R=366$, two more vortices drop out, and the resulting state of 22 cells and five waves then lies in the operating space of figure 7. An instance of an unnatural trajectory is also provided by this same figure. By changing the speed of the inner cylinder sufficiently rapidly, the state can be made to jump to right or left across the gap between $R=407$ and $R=482$ in the curve for five waves, or even across the gap between $R=201$ and $R=679$ in the curve for four waves. In both of these examples, of course, the same final states are also accessible through slow changes in speed alone. 
an appreciation of the gradually emerging pattern of transitions, and several permissible operating states were in fact found by being deliberately sought in certain ranges of speed.

\subsection{The concept of a mean state}

The structural details of the three-dimensional representation in figure 8 will obviously vary from one experimental situation to another. This figure is only a single section through a much more complicated figure incorporating the several additional dimensions needed to describe the effects of changes in geometry (e.g. gap/radius ratio or length/gap ratio), changes in noise level (cf. the influence of end conditions), steady rotation of the outer cylinder in either direction, and so on. The best hope for a successful interpretation of the

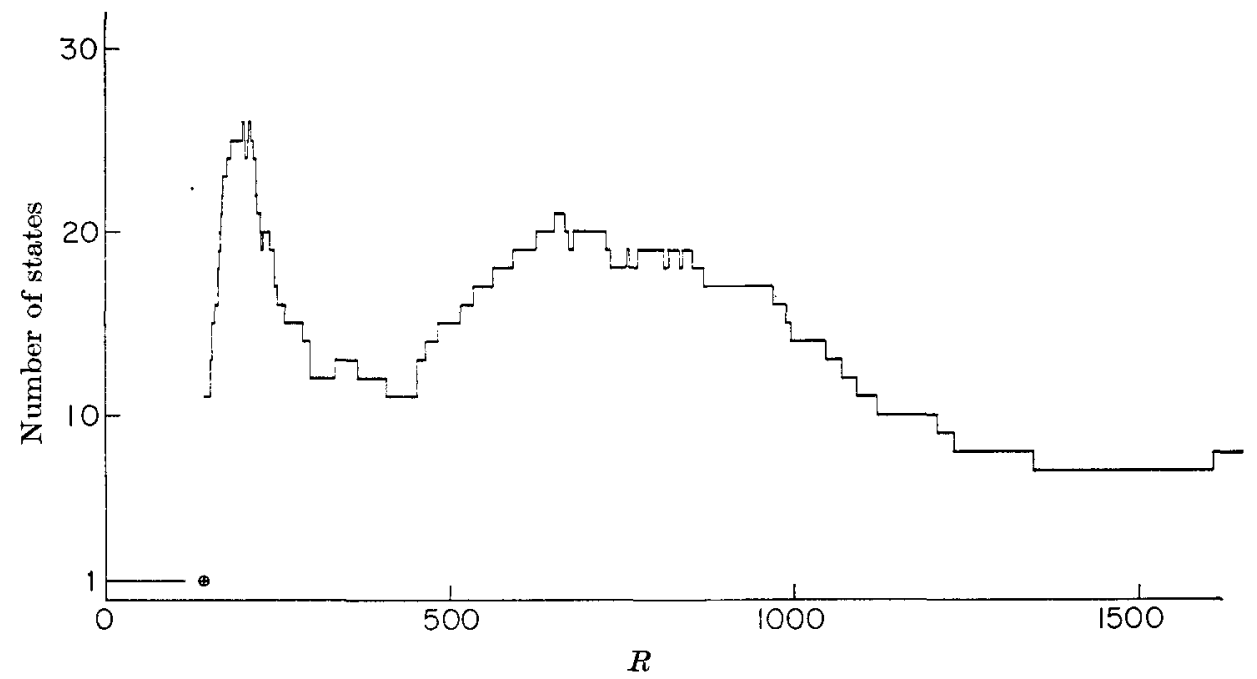

FIGURE 9. Number of accessible states as a function of speed (precision cylinders; $\left.r_{o} / r_{i}=1 \cdot 144\right)$. Reynolds number $R$ is based on gap and on surface speed of inner cylinder.

data therefore lies not in a study of fine detail in figure 8, but in a study of the general topological properties of the figure as a typical collection of trajectories in wave-number space.

The experimental fact is that the steady Couette flow of a given fluid in a given apparatus is not uniquely determined by the speed of rotation, and that the issue of uniqueness raised by this finding is of a different order from the issue of uniqueness usually at stake in discussions of stability. As shown in figure 9, at any specified speed in the doubly-periodic régime there exists a variety of possible operating states, sometimes 20 or more in number, among which the one actually observed is determined by the whole previous history of the experiment. In figure 10, the distribution of these accessible states in wave-number space is examined for several Reynolds numbers. The solid points denote states which are definitely stable, in the sense that the flow is permanent as long as the speed is held constant. The open points denote states which are definitely unstable; i.e. they can be observed, if at all, only momentarily during the transient phase following a 
rapid adjustment of the speed to the value in question, and are then replaced by some other state. The solid lines, derived in part by interpolation in figure 8 , denote a hypothetical continuous boundary between the stable and unstable regions.

It is clear in figure 10 that the accessible region in wave-number space depends on Reynolds number. In order to obtain a measure of this dependence, the ensemble of accessible states at each speed can be replaced by a fictitious mean state defined by taking the average wave-numbers over the states actually observed. The result lies as shown by the quartered circles in the figure. Because this concept of a mean state may be a meaningful abstraction in any comparison of one experiment with another experiment or with theory, mean wave-numbers have been computed for all of the various intervals between state transitions and entered in table 1. The same quantities are also plotted against Reynolds number in figure 11.

Unfortunately, it is also clear in figure 10 that the data are defective in certain ranges of Reynolds number, in that the boundary between the stable and unstable regions is not completely defined. In the range $R=250-450$, for example, a few states with eight waves probably should have been uncovered. $\dagger$ In the range $R=800-1300$, one or more additional high-wave-number states, and especially the state $30 / 5$, are almost certainly stable. In this same range $R=800-1300$, finally, there is good reason to believe that several permissible states with 16 or even 14 cells should have been observed, except that mechanical limitations on the apparatus prevented the manipulation of speed or acceleration necessary for their establishment. $\ddagger$

To illustrate the evolution of the doubly-periodic Couette flow with increasing speed, figure 19 (plates 6 and 7 ), shows a sequence of six photographs taken at the circled points marked 19a, $19 \mathrm{~b}$, etc., in figure 11 . The state in each case is as close as possible to the mean state just defined. The last two photographs, in particular, show how transition to turbulence begins in this apparatus at $R \sim 1300$ with a gradual emergence of noise in the form of a gradually increasing irregularity of the flow.

At each of three Reynolds numbers $R \sim 455,785$, and 1040, the physical appearance of two extreme states far from the mean state is shown in figure 20

† The usual experimental method for establishing a flow with a high tangential wavenumber was to approach the final operating condition from the singly-periodic side with the two cylinders rotating initially in the same direction. A sufficiently fast stop for the outer cylinder then usually led to a flow with a large number of tangential waves, probably as a result of Tollmien instability in the unsteady viscous layer on the wall of the outer cylinder (cf. figure 22 (o), plate 12).

† The usual experimental method for establishing a flow with a low axial wave number was to run the inner cylinder at the highest practicable speed, and then to run the outer cylinder briefly in the opposite direction to obtain an irregular turbulent motion. When the outer cylinder was stopped, the resulting flow was usually one with 20 or 18 cells. States with 16 or 14 cells, however, could not be achieved by this method. Further increases in speed for the inner cylinder were not thought to be mechanically safe, and a reduction of the viscosity to increase the Reynolds number would have modified the whole experiment by introducing new problems with speed regulation and thermal convection near the Taylor boundary. 
(plates 8 and 9), in order to demonstrate the remarkable differences in flow configuration which can be observed at a given speed. Among these photographs, which correspond to the joined pairs of points marked $20 \mathrm{a}, 20 \mathrm{~b}$, etc., in figure 11 , the one at the point $20 \mathrm{c}$ is noteworthy on account of a subharmonic disturbance in the axial direction, a disturbance which is repeated in four cells rather than two.
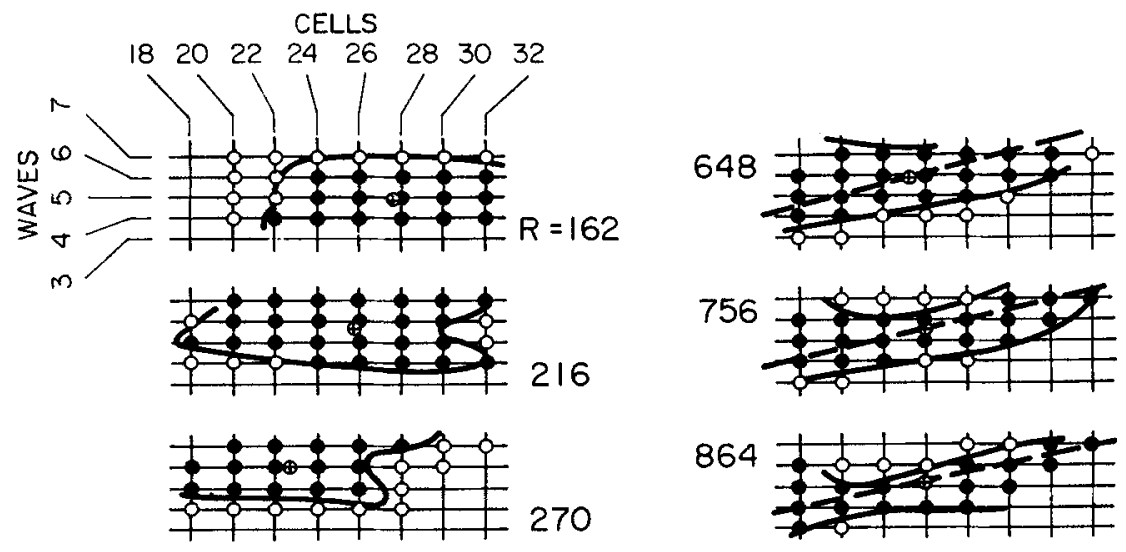

864
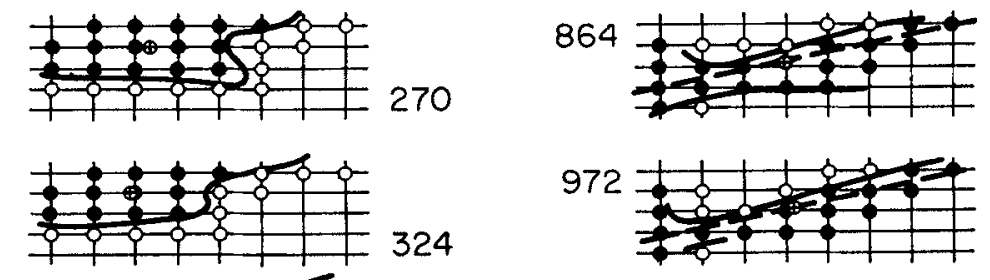

972
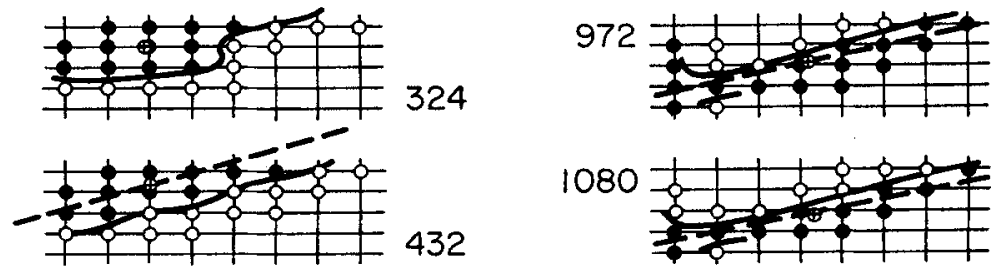

432
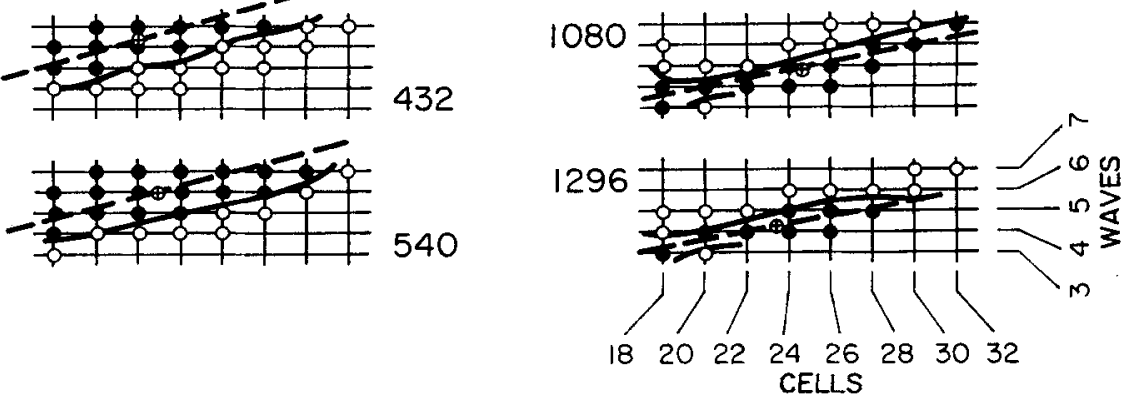

Figure 10. Accessible states (filled circles) and inaccessible states (open circles) in wavenumber space at various Reynolds numbers $R$ based on gap and on surface speed of inner cylinder (precision cylinders; $r_{o} / r_{i}=1 \cdot 144$ ). Quartered circles show position of mean state, defined as centre of gravity for observed population of accessible states. Dashed lines show constant ratio of axial to tangential wave-number.

Finally, a representative group of photographs taken at the points marked $21 \mathrm{a}, 2 \mathrm{Ib}$, etc., in figure 11 is presented in figure 21 (plates 10 and 11), to illustrate some further experimental observations. At the point 21 a there is a welldeveloped second harmonic in the tangential direction. At the point $21 \mathrm{~b}$ there is a complicated but quite well correlated pattern at a moderately high Reynolds number. The flow at the point $21 \mathrm{c}$ shows, near the centre of the apparatus, a striking example of the kind of disturbance which characterizes the first appearance of noise. At the point $12 \mathrm{~d}$ there is a marked distortion or nonuniformity in the axial direction, indicating the presence of strain and the 
imminence of a change in state. In this case the pressure on the flow pattern is actually relieved by an increase in the number of tangential waves from four to five, the number of cells remaining unchanged but the spacing becoming much more regular.

The photograph at the point 21 e shows a flow undergoing just such a transition, with the number of tangential waves changing from four to five. The physical process involved is that the tangential waves first become gradually unequal in
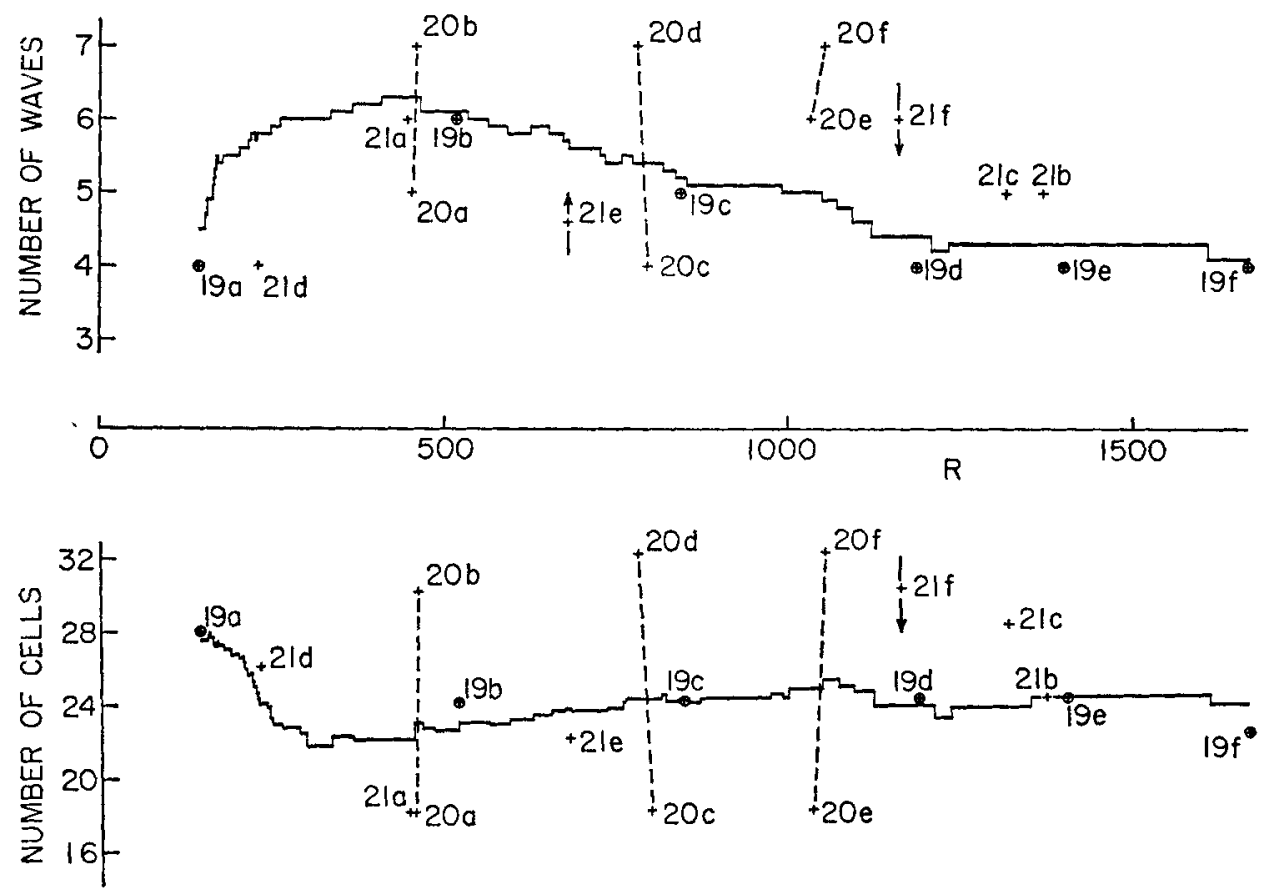

Frgure 11. Expected (mean) values for axial and tangential wave-numbers as a function of speed (precision cylinders; $r_{o} / r_{i}=1 \cdot 144$ ). Reynolds number $R$ is based on gap and on surface speed of inner cylinder. For photographs of flow at points $19 \mathrm{a}, 19 \mathrm{~b}$, etc., see figures 19, 20 and 21 (plates 6-11).

wavelength and amplitude; a long wave may then change into two, or a short wave may disappear, and the pattern then becomes once more very regular. This process sometimes begins at one end of the apparatus and proceeds toward the other, as in the photograph, but it can also occur uniformly and with perfect phase along the whole length of the working space. Certain of these transitions in tangential wave-number forecast their coming by inducing the strong axial non-uniformity already illustrated in figure $21(\mathrm{~d})$, (plate 11), and others do so by way of a strong beat in the optical signal whenever the change in the pattern is relatively slight during one revolution of the distorted tangential wave pattern. This phenomenon of beating, incidentally is useful in providing an automatic check on the number of waves.

The last photograph in plate 11, at the point marked $21 \mathrm{f}$ in figure 11 , shows the flow during a transition in which the number of cells is decreasing from 
30 to 28 . Two adjacent cells have apparently merged at one point, forming an elongated loop (similar to the one in figure 15 (d), plate 2 , but much less regular) which rapidly shrinks in length and disappears while the rest of the pattern expands into the space thus made available. Such changes are often encountered in fairly violent transitions, while less violent ones seem to involve only an axially symmetric collapse of a vortex pair. Transitions of both types also forecast their coming. Reference to the first shadowgraph in plate 1 shows that adjacent vortices are normally separated by partitions which are alternately in and out of phase along the length of the apparatus. The first sign of weakness in a vortex pair which is about to drop out during a state transition is a shift of the internal partition into phase with its two neighbours, and this is followed by local damping of the tangential wave motion and finally by collapse of the two vortices into each other, symmetrically or otherwise. The whole process is essentially a rapid traverse backwards through the same changes in flow structure which occurred when the doubly-periodic flow was originally established.

\subsection{The concept of a preferred or expected state}

So far, the concept of a mean state has been treated entirely as a static concept requiring no special information about the nature of the individual state transitions. As it happens, this concept can also be interpreted dynamically. To begin with, the trajectory of the mean state through the space of the state diagram, figure 8 , is a skewed curve whose projections on the vertical co-ordinate planes have been given in figure 11 . If now the various levels of the state diagram are moved horizontally so as to make the mean-state curve a vertical straight line, the skewness is transferred to the trajectories for fixed wave-number. Several views of the resulting surface for the case of six waves are shown in figure 12. The filled arrows in the top and bottom views indicate the direction of the local trajectory (with respect to the mean state) when each transition occurs, and the arrows drawn in outline indicate the direction and magnitude of the transition itself. With the aid of this and similar figures which can be drawn for the remaining data, it can be shown that for an overwhelming majority of the 74 state transitions the initial trajectory in wave-number space is moving definitely away from the mean or preferred state just before the transition occurs, and for a large if not overwhelming majority of cases (60 out of 74) $\dagger$ the transition itself is definitely toward the mean or preferred state.

At two points along the lower boundary of the surface depicted in figure 12, the number of waves remains the same during the transition, but the number of cells increases by four. Transitions of this type are quite spectacular. What is observed is always the sudden appearance of four vortices in two pairs, with one pair springing into view between the first and second vortex at each end of the working space. This phenomenon can be qualitatively understood with the aid of figure 13, in which the co-ordinates are Reynolds number and axial wave-

$\dagger$ The exceptions seem for the most part to point toward the area of low axial wave numbers at high speeds in figure 10, where mechanical problems may have prevented the realization of certain possible states. As a consequence of this failure, the mean state has probably been misplaced in this range of Reynolds number. 
number, both normalized to unity at the Taylor boundary in order to suppress some of the effects of gap/radius ratio. When the condition of neutral stability is applied in the linearized problem of axially symmetric disturbances, the

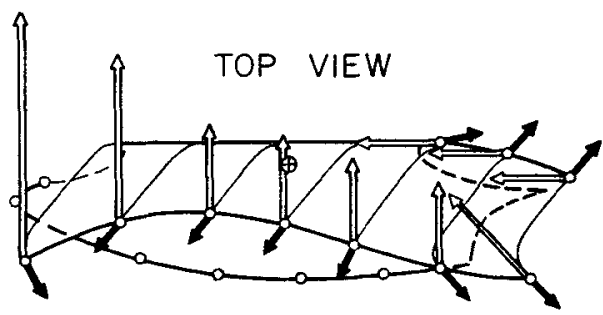

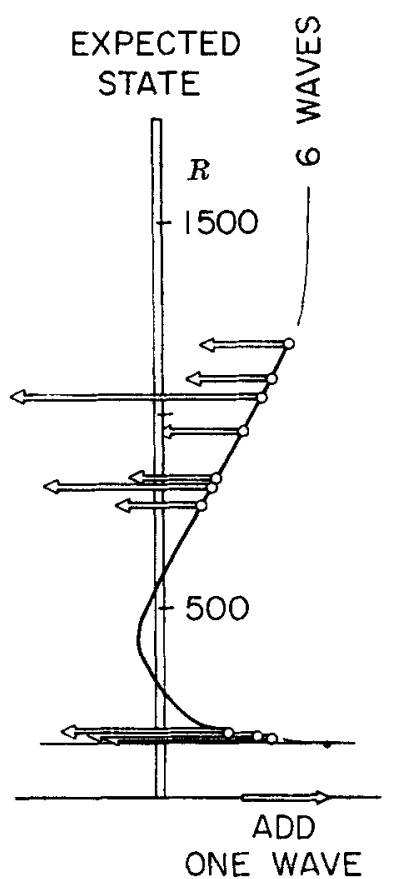

SIDE VIEW

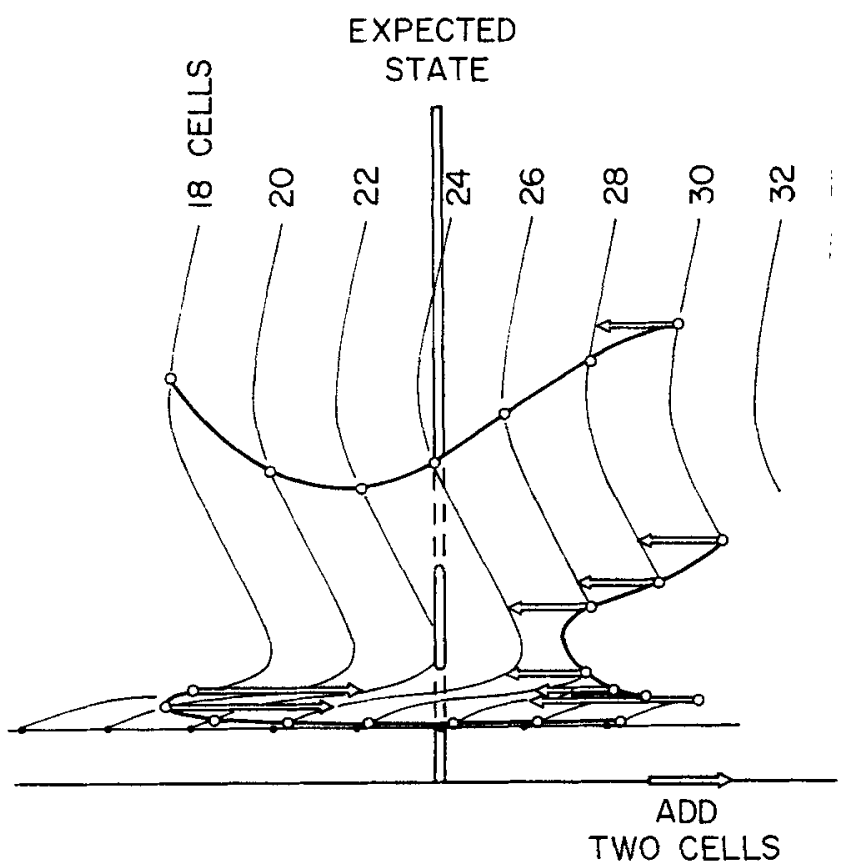

FRONT VIEW

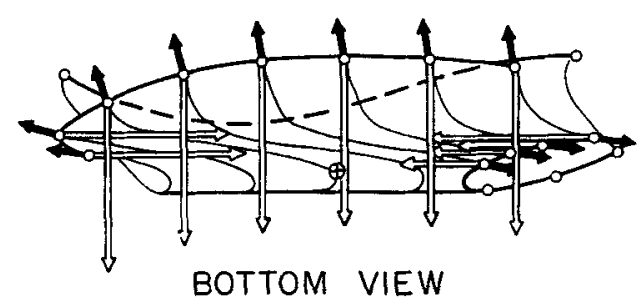

Figdre 12. Accessible region and state transitions for case of 6 tangential waves with outer cylinder at rest (precision cylinders; $r_{o} / r_{i}=1 \cdot 144$ ). Vertical co-ordinate $R$ is: Reynolds number based on gap and on surface speed of inner cylinder. Filled arrows in top and bottom views show trajectory with respect to mean state before transition; open arrows show change of state during transition. 
relationship between wave-number and speed is a curve which is very slightly concave upward. $\dagger$ For the least value of the critical Reynolds number, which is the only relevant one when the speed increases monotonically from zero, the cell cross-section is very nearly square. However, the experimental situation can easily be arranged so that this same stability boundary is approached from above, with the speed of the inner cylinder decreasing, while the axial wave-number is abnormally small. Under these conditions, a speed must eventually be reached

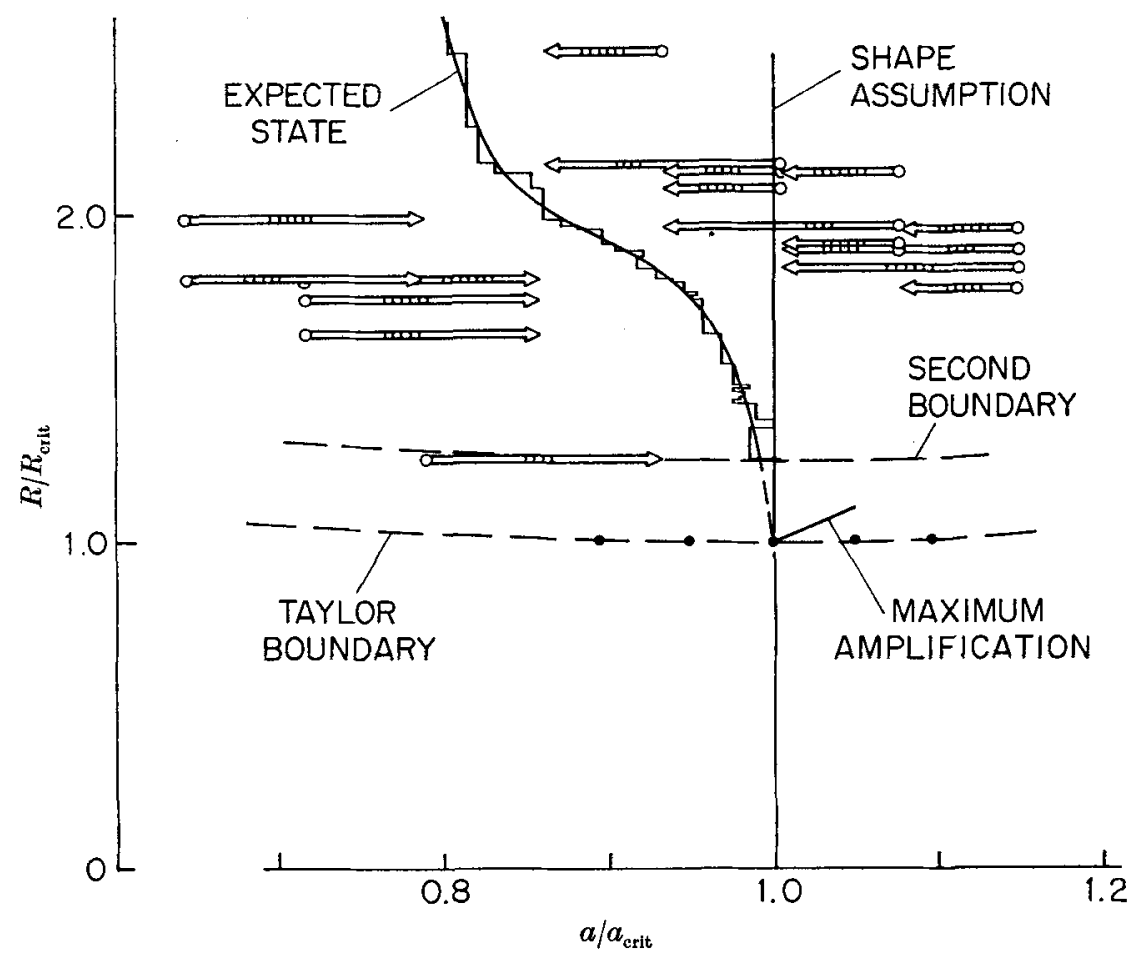

Figure 13. Normalized trajectories in axial wave-number space above the Taylor boundary. Axial wave-number $a=2 \pi\left(r_{0}-r_{i}\right) / \lambda$ where $\lambda$ is axial wave length. 'Maximum amplification' curve is computed from linearized theory for $r_{o} / r_{i}=1 \cdot 111$ (Donnelly; private communication). 'Expected state' curve is taken from present experiments for $r_{o} / r_{i}=1 \cdot 144$ (cf. figure 11). Arrows show spontaneous state transitions for slowly decreasing speed (left side of figure) or slowly increasing speed (right side of figure). Bars on arrows indicate number of tangential waves.

at which the flow is no longer unstable for the wavelength actually present. The cells cannot disappear, however, as long as the flow is still unstable for a cell structure which is more nearly square. Thus it is not surprising in an apparatus of fixed dimensions to observe the abrupt appearance of new cells. For the experimental apparatus used here, this phenomenon occurs as indicated by the arrows at the left in figure 13. With one marginal exception, such transitions

$\dagger$ The curve in the figure reproduces Taylor's original calculation for the limiting case $r_{o} / r_{i} \rightarrow 1$, extrapolated outside the original range of definition by fitting a parabola to the graph on p. 311 of Taylor's 1923 paper. 
actually belong to the doubly-periodic rather than the singly-periodic flow régime.

It was entirely a matter of luck that the GALCIT Couette-flow apparatus was made short enough to allow a reasonably complete survey of the state problem, but not so short that the mean data suffer from excessive dispersion. If similar experiments were to be carried out in a much longer but still finite apparatus, however, with a proportionately greater number of state transitions in the same range of speed, the effort required to maintain a deterministic point of view might be prohibitive. Even in the case of the present measurements, it can be argued that a completely deterministic point of view toward the experiment in the case of steady flow is already almost untenable, even though the motion itself is laminar by every ordinary standard. The central experimental difficulty is that an observer who knows the current steady operating conditions for the apparatus (geometry, fluid properties, speed), but does not know the previous operating history, will be unable to specify the two characteristic wave-numbers which are needed to determine the flow in detail. In principle, of course, a sufficiently accurate knowledge of the previous history should be enough to remove the indeterminacy; and in practice a skilled operator can usually-but not alwaysproduce on demand a specified flow at a specified permissible speed. The occasional failures, however, and the prospect of much greater difficulties with a much longer apparatus, are significant. They suggest that the phenomenon is already complicated enough to allow, and perhaps to require, an appeal to the doctrine of indeterminacy. According to this doctrine, when the result of a single observation of an event is controlled by factors some of which are unknown, or difficult to measure, or capable of producing disproportionately large effects, it is customary to say that the result depends on chance. An application of this doctrine usually requires an analysis of the results of a large number of observations in terms of relative frequency, and the analysis is considered to be successful if it yields a plausible and well-defined probability structure for the result.

In the present context, the implication is that statistically generated data might be used to define an expected state for the flow in the usual statistical sense as the position of the centre of gravity for a large number of results distributed in wave-number space according to their relative frequency. Although it may not be practical to conduct a real experiment in this manner, either because of bias on the part of the operator or because of mechanical limitations, the problem could in principle be approached analytically as a random-walk process (for a slowly changing speed of the inner cylinder, say) punctuated by occasional encounters with state transitions of known type and position. At any rate, a strong impression was gained during the experiments that the mechanical difficulty in achieving a particular state increased rapidly, so that presumably the probability of achieving this same state by random means decreased rapidly, as the state in question became more remote from the mean state already defined. Finally, it has already been pointed out that the concept of a mean state has a dynamic significance; the flow tends to resist large excursions from the mean or preferred state and to respond by a spontaneous return toward it through a state transition. For all of these reasons, it is not obvious that there is any significant. 

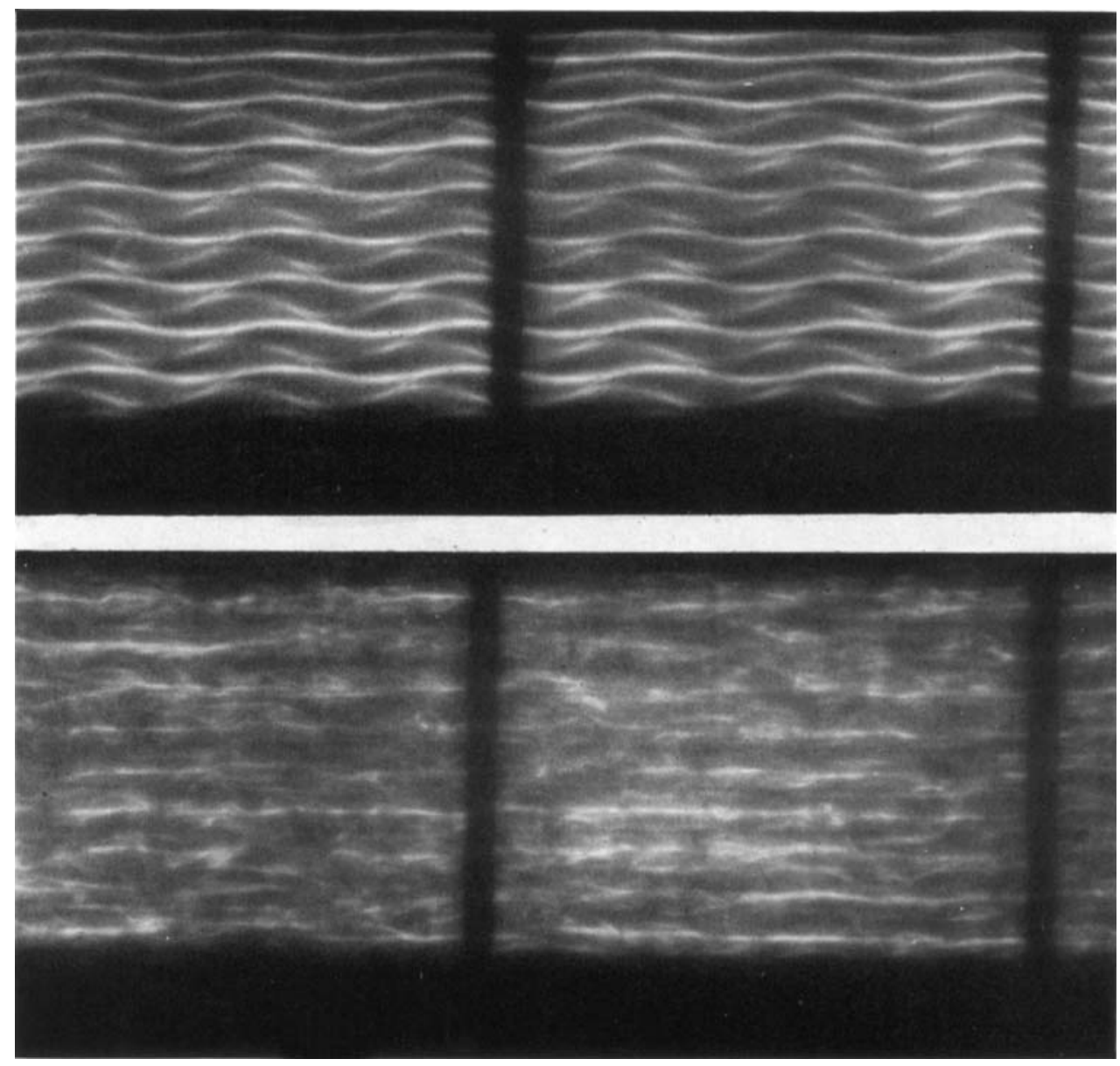

Figure 14. Shadowgraphs of circular Couette flow (precision cylinders; $r_{o} / r_{i}=1 \cdot 144$ ). Light source is baffled xenon flash lamp on axis of rotation. Upper photograph shows doubly-periodic laminar flow (24 cells, 6 waves) for $R_{i}=\omega_{i} r_{i} / \nu=3700, R_{o}=\omega_{0} r_{o}^{2} / \nu=0$. Lower photograph shows turbulent flow with residual axial periodicity at $R_{i}=9200$, $R_{o}=-11,900$. 

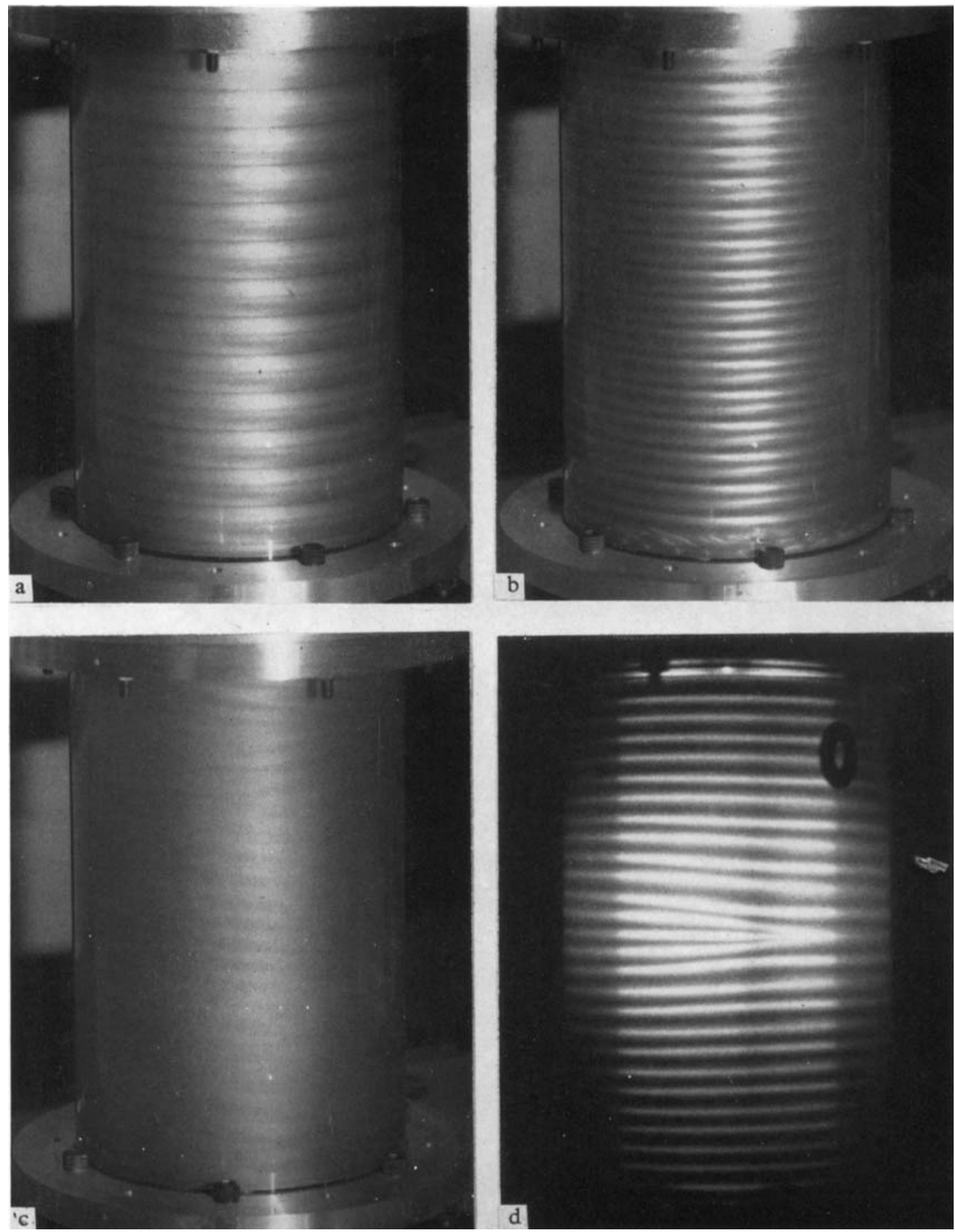

Figure 15. Singly periodic laminar Couette flows near the Taylor stability boundary (rough cylinders; $r_{o} / r_{i}=1 \cdot 135$ ). Light source for (a), (b), (c) is spark gap; scene in (d) is takken from motion picture. (a) Flow at $\mathrm{A}$ in figures 2 (b) and (c); $R_{i}=830, R_{o}=0$. (b) Flow at $\mathrm{B}$ in figure $2(\mathrm{~b}) ; R_{i}=8090, R_{0}=7350$. (c) Flow at $\mathrm{C}$ in figure $2(\mathrm{~b}) ; R_{i}=3820$, $R_{o}=-12,900$. (d) Flow at D in figure $2(\mathrm{c}) ; R_{i}=1780, R_{o}=560$.

COLES 

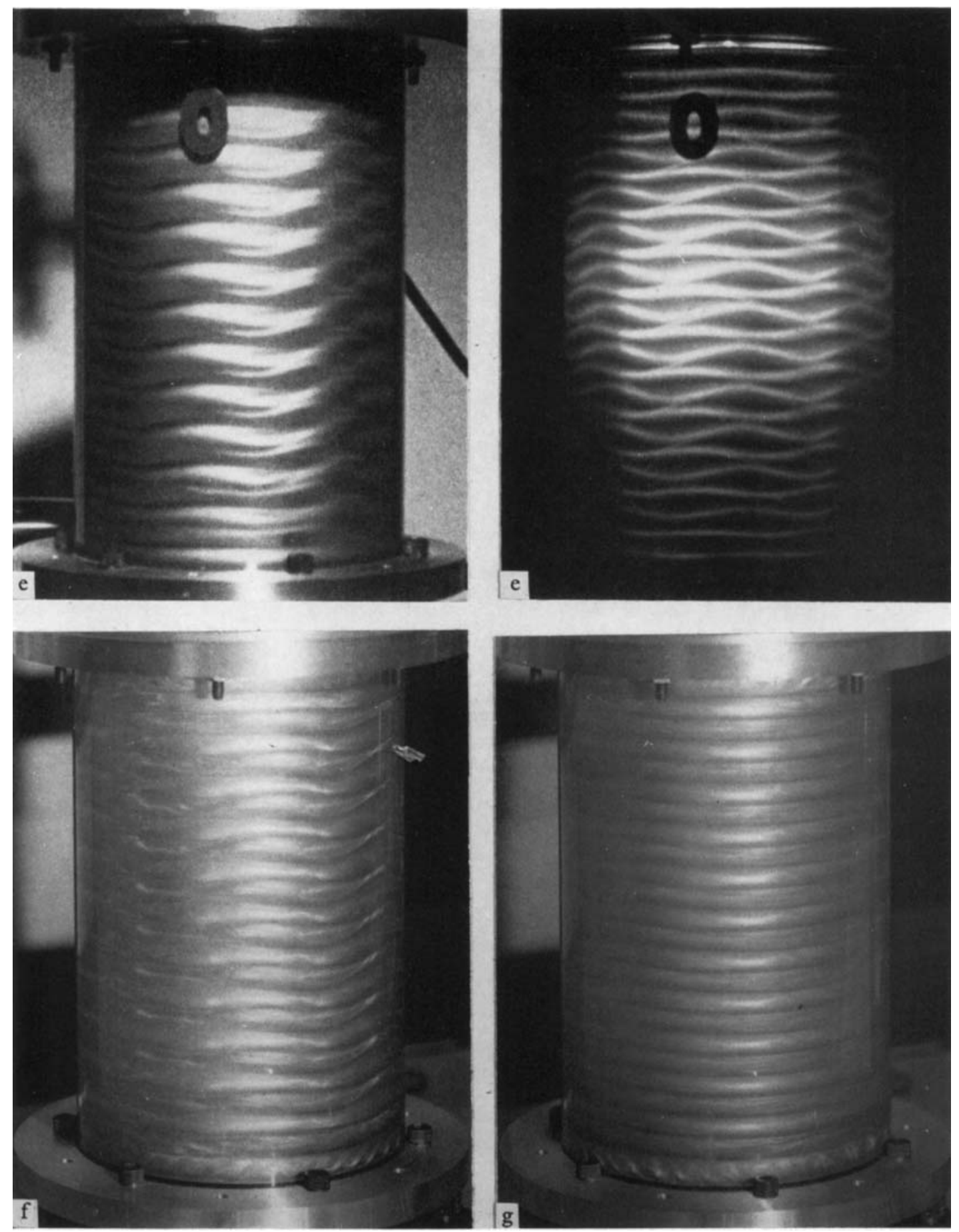

Figure 16. Doubly-periodic laminar Couette flows above the second stability boundary (rough cylinders; $r_{o} / r_{i}=1 \cdot 135$ ). Scenes in (e) are taken from motion picture; light source for (f) and (g) is spark gap. (e) Flow at $\mathrm{E}$ in figure 2 (c), front-lighted and back-lighted; $R_{i}=1800, R_{o}=0$. (f) Flow at $\mathrm{F}$ in figures 2 (a) and (b); $R_{i}=7440, R_{o}=0$. (g) Flow at $\mathrm{G}$ in figure $2(\mathrm{~b}) ; R_{i}=8050, R_{0}=2280$. 

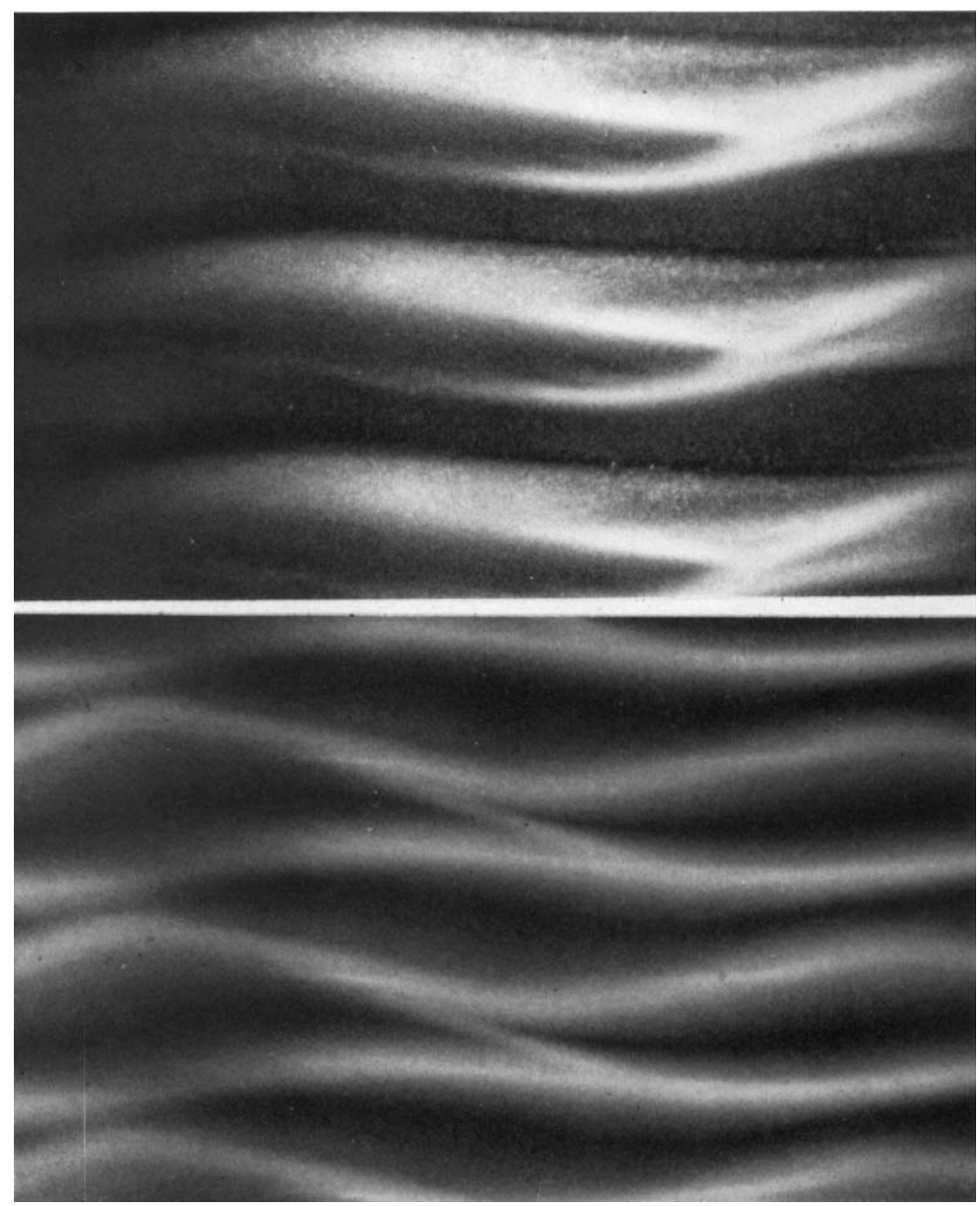

FIGURJs 17. Close-up views of doubly-periodic laminar Couette flow at $\mathrm{E}$ in figure 2 (c), front-lighted and back-lighted (rough cylinders; $r_{o} / r_{i}=1 \cdot 135$ ). Scenes taken from motion pieture. $R_{i}=1800 ; R_{o}=0$. 

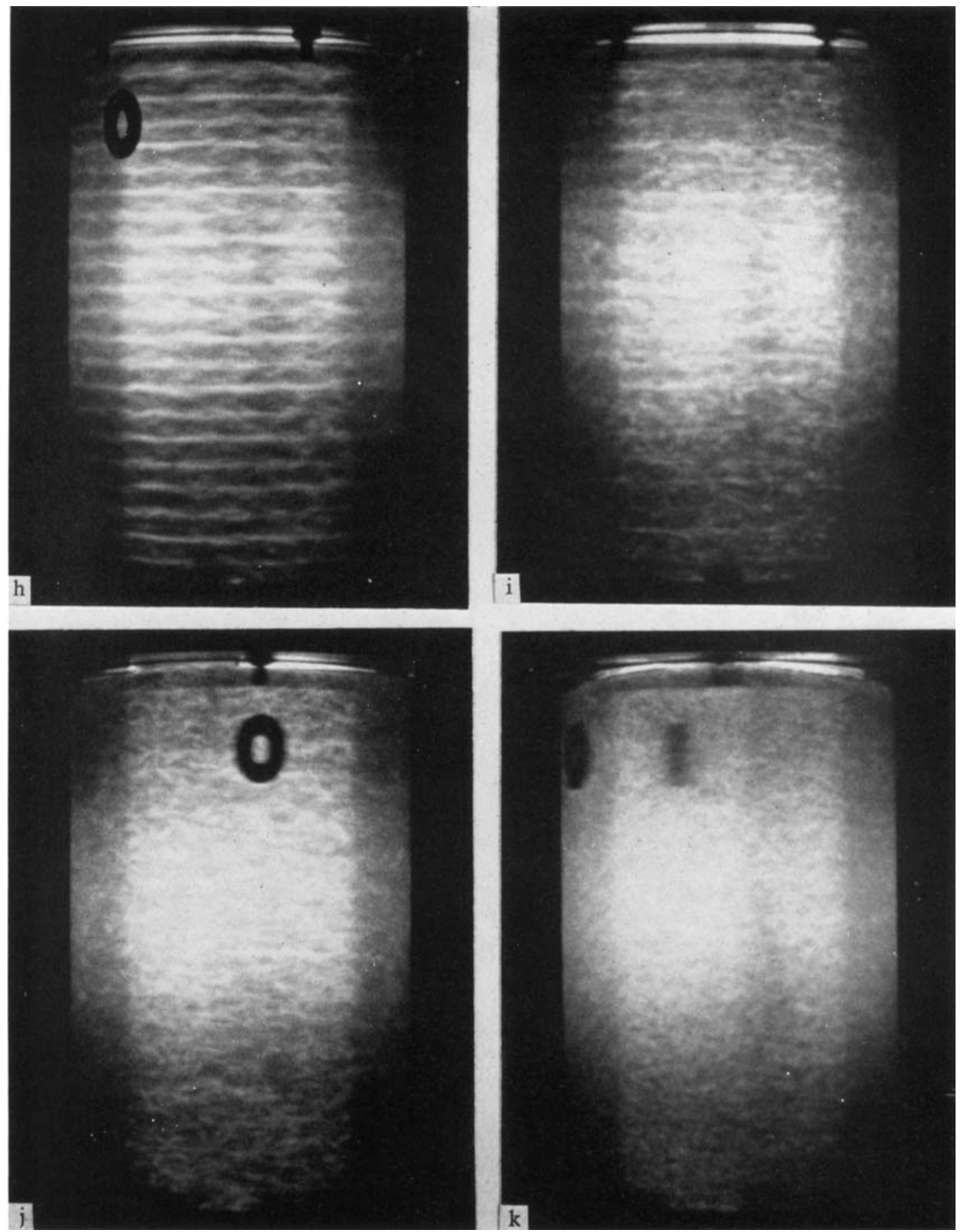

Figure 18. Turbulent Couette flows showing disappearance of residual periodicity when outer cylinder rotates opposite to inner one (rough cylinders; $r_{o} / r_{i}=1 \cdot 135$ ). All scenes are taken from motion picture. (h) Flow at $\mathrm{H}$ in figure $2(\mathrm{a}) ; R_{i}=18,800, R_{0}=0$. (i) Flow at I in figure $2(\mathrm{a}) ; R_{i}=18,800, R_{o}=-10,850$. (j) Flow at J in figures 2 (a) and (b); $R_{i}=8350$, $R_{\mathrm{s}}=-10,850$. (k) Flow at $\mathrm{K}$ in figure $2(\mathrm{a}) ; R_{i}=18,900, R_{0}=-27,700$.

\section{COLES}


Journal of Fluid Mechanics, Vol. 21, part 3

Plate 6
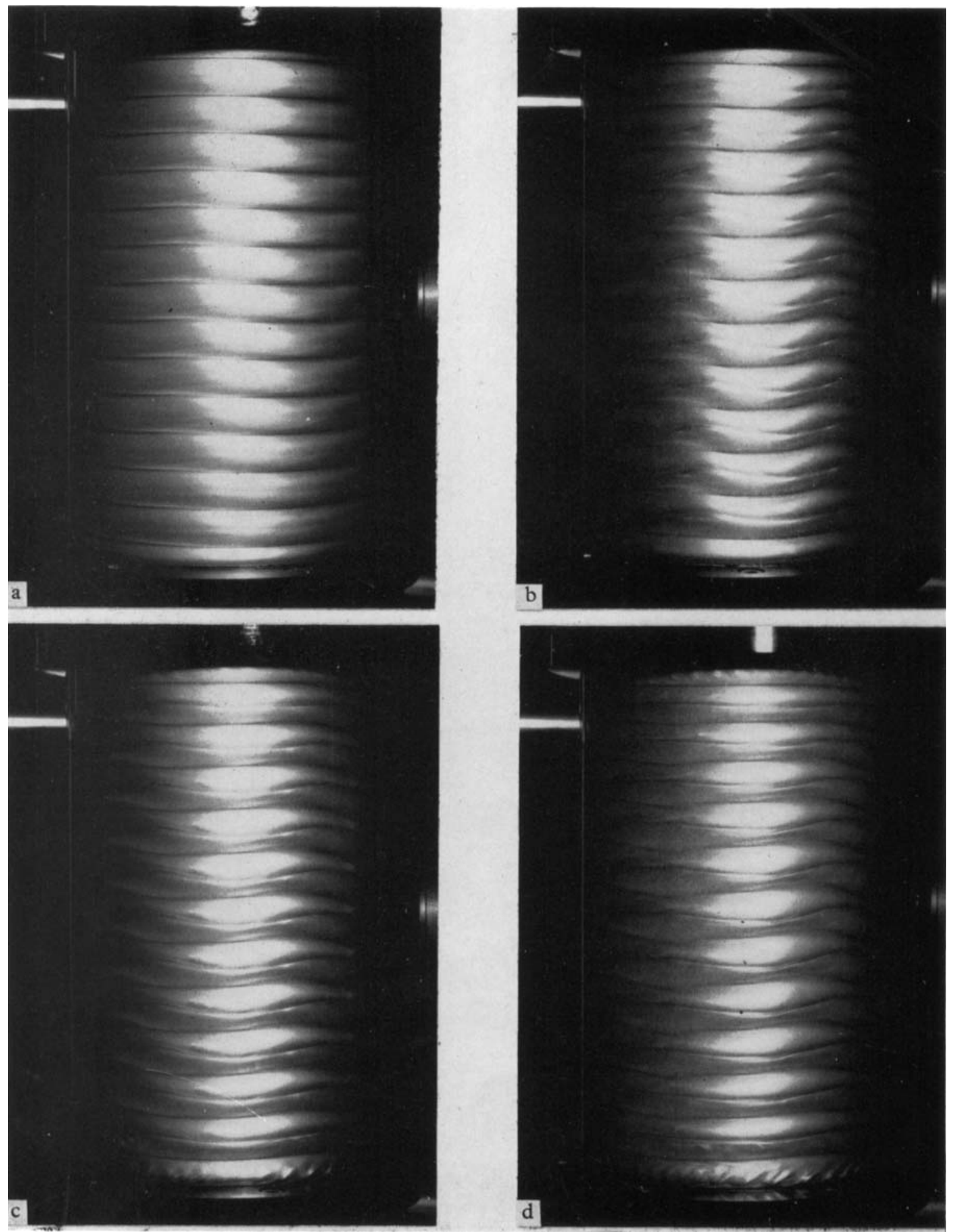

\section{COLES}

Figure 19. For legend see facing page. 

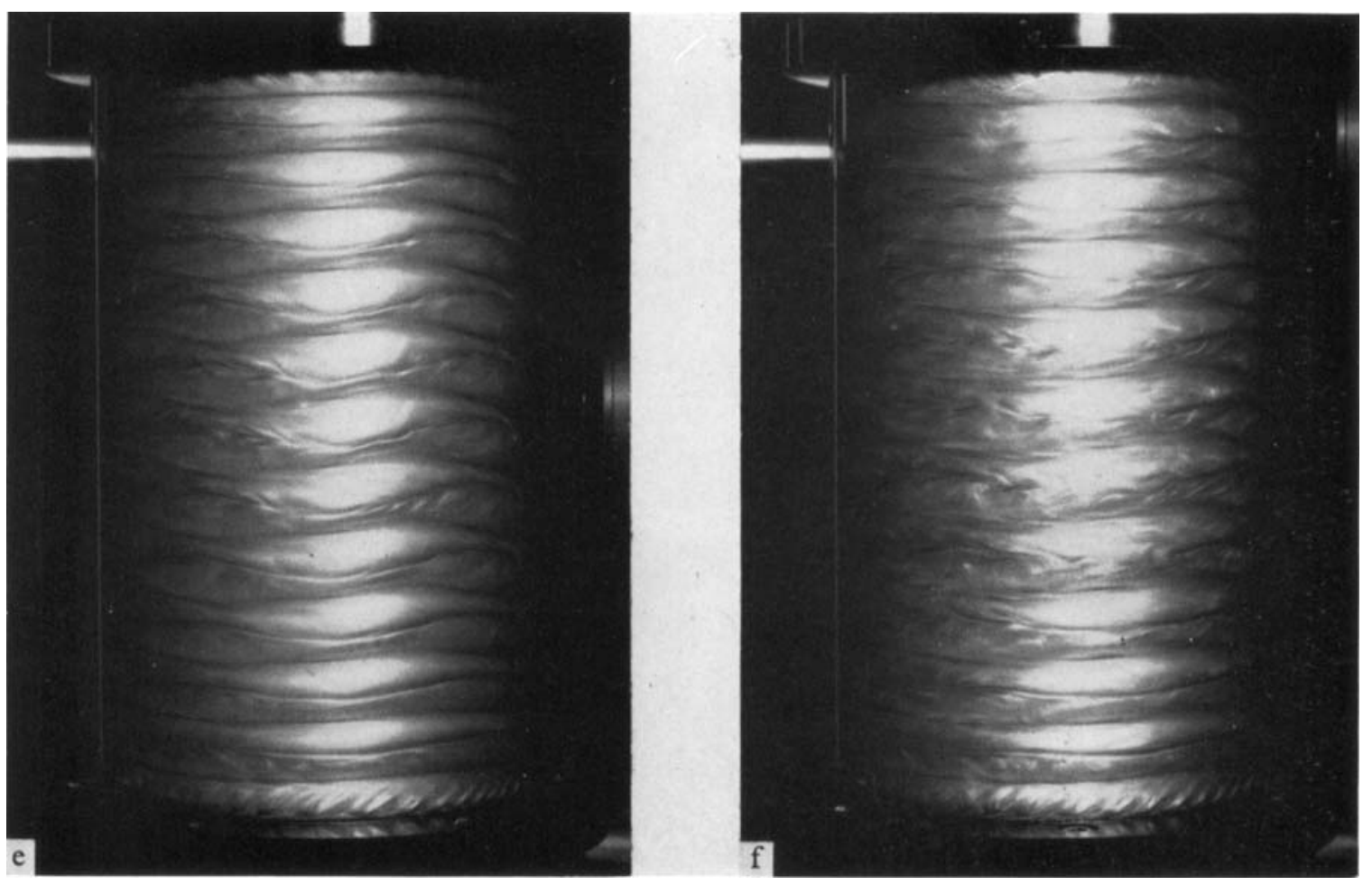

Figure 19. Development of doubly-periodic Couette flow with speed when flow is close to expected state (precision cylinders; $r_{o} / r_{i}=1 \cdot 144$ ). Note appearance of end disturbance between (b) and (c) at $R \sim 690$. Note also first appearance of randomness between (d) and (e) at $R \sim 1300$. Operating states in figure 11 are denoted by points numbered $6 \mathrm{a}, 6 \mathrm{~b}$, etc., to correspond to photos. Reynolds number $R$ is based on gap and surface speed of inner cylinder.

(a) Photo. no. $35, R=143$, state $28 / 4$.

(b) Photo. no. 46, $R=515$, state $24 / 6$.

(c) Photo. no. 42, $R=842$, state $24 / 5$.

(d) Photo. no. 127, $R=1185$, state $24 / 4$.

(e) Photo. no. 126, $R=1400$, state $24 / 4$.

(f) Photo. no. 150, $R=1665$, state $22 / 4$. 


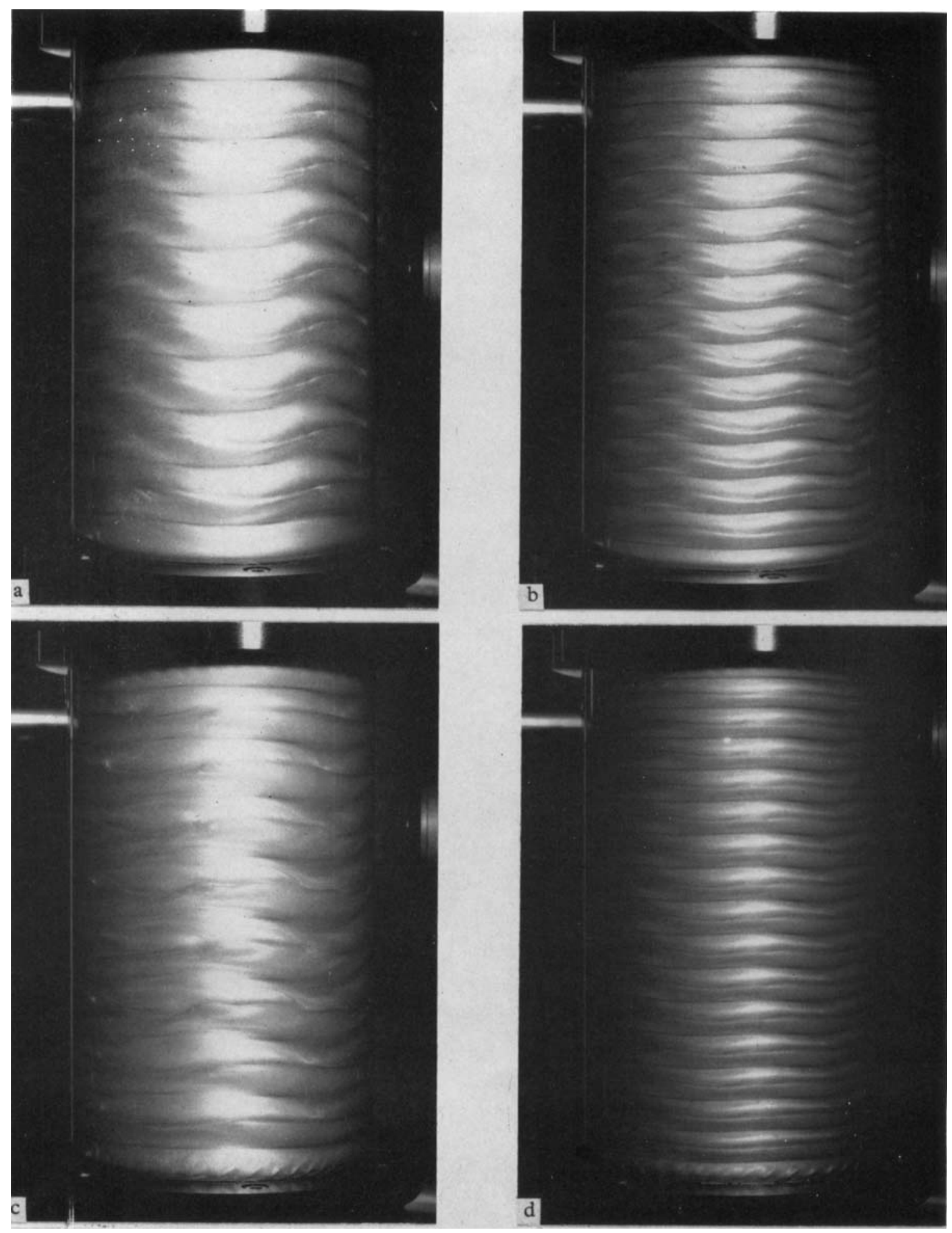

Figure 20. For legend see facing page. 

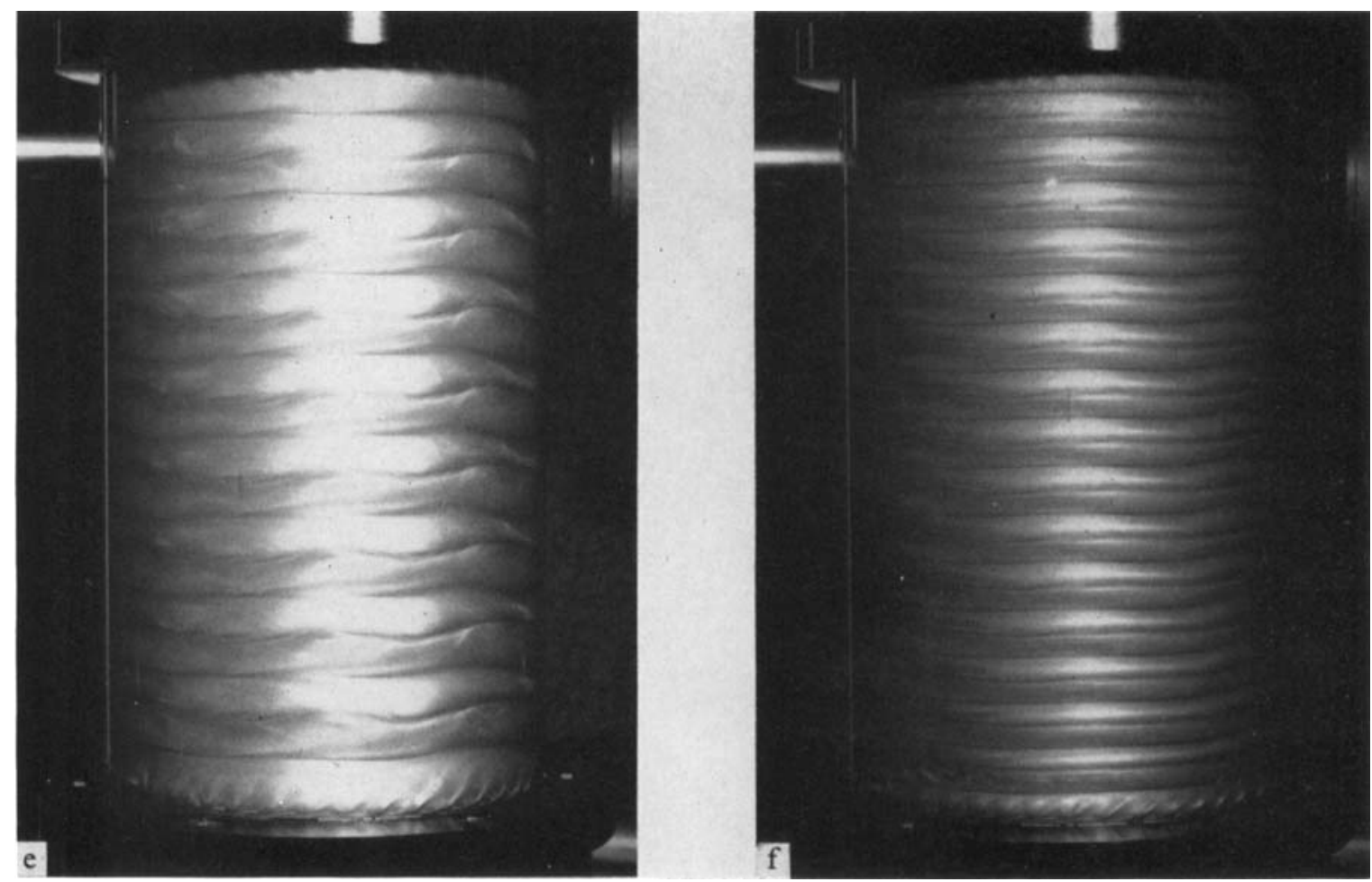

Figure 20. Comparison of extreme states in doubly periodic Couette flow at fixed Reynolds number (precision cylinders; $r_{o} / r_{i}=1 \cdot 144$ ). Operating states in figure 11 are denoted by joined pairs of points numbered $7 \mathrm{a}, 7 \mathrm{~b}$, etc., to correspond to photos. Reynolds number $R$ is based on gap and surface speed of inner cylinder. Further comments: (a), (b) State 30/7 in (b) changes to $28 / 7$ at $R=419$ (speed decreasing). (c), (d) Note axial subharmonic in (c). State $32 / 7$ in (d) changes violently to $28 / 6$ at $R=756$ (speed decreasing). (e). (f) State $18 / 6$ in (e) changes to $18 / 3$ at $R=1046$ (speed increasing). State $32 / 7$ in (f) changes violently to $26 / 4$ at $R=1119$ (speed increasing).
(a) Photo. no. 144, $R=453$, state $18 / 5$.
(b) Photo. no. 136, $R=459$, state $30 / 7$.
(c) Photo. no. $142, R=794$, state $18 / 4$.
(d) Photo. no. $157, R=778$, state $32 / 7$.
(e) Photo. no. 147, $R=1030$, state $18 / 6$.
(f) Photo. no. 156, $R=1051$, state $32 / 7$. 

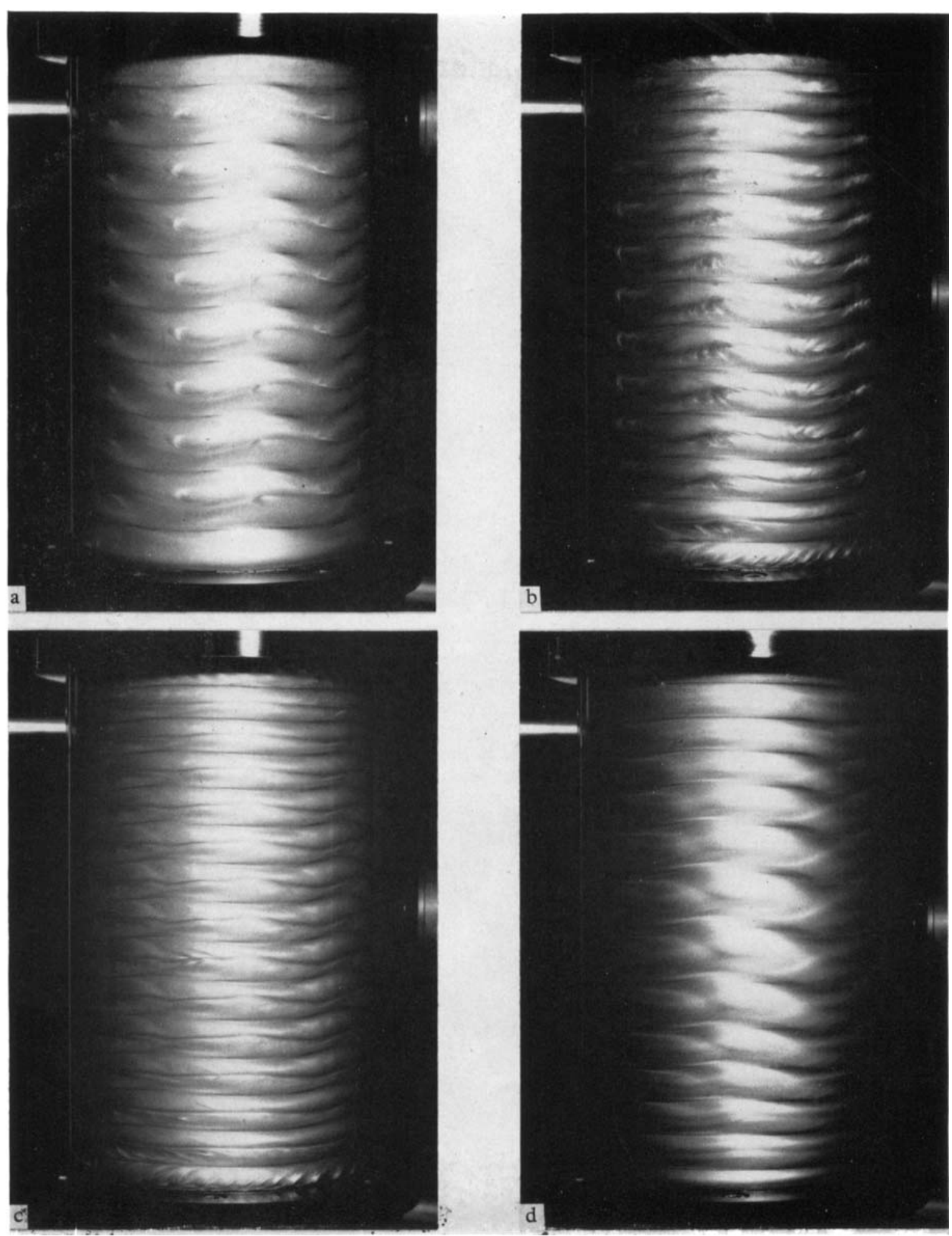

Figure 21. For legend see facing page. 

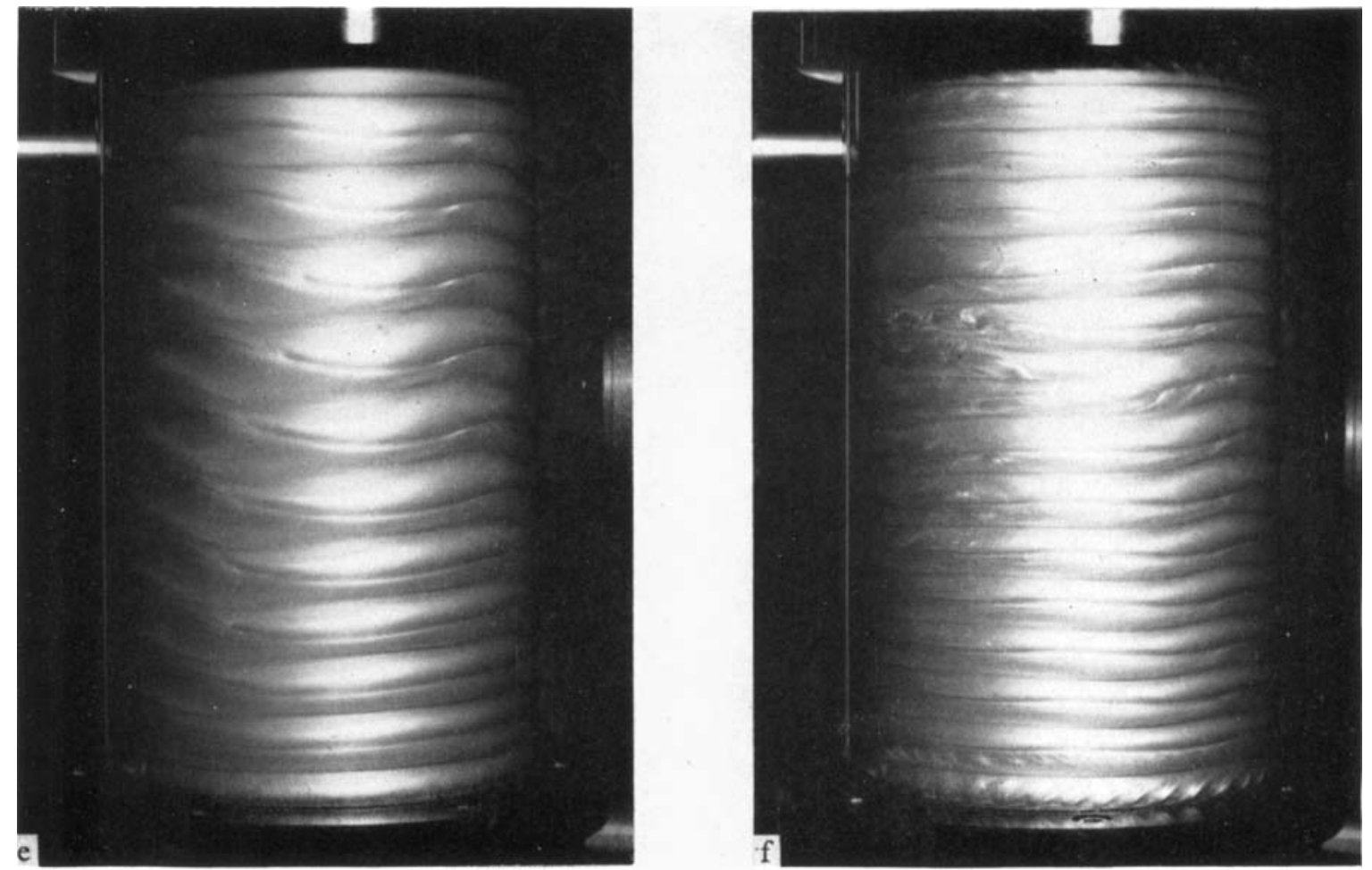

Figure 21. Details of several phenomena in doubly-periodic Couette flow (precision cylinders; $r_{o} / r_{i}=1 \cdot 144$ ). Operating states in figure 11 are denoted by points numbered $8 \mathrm{a}, 8 \mathrm{~b}$, etc., to correspond to photos. Reynolds number $R$ is based on gap and surface speed of inner cylinder. Further comments: (a) Note strong tangential second harmonic. (b) Highly correlated laminar flow; compare figure 19 (e) (plate 7), for expected flow at same Reynolds number. Data book says 'quite unsteady'. (c) Note quantum of noise near centre of working space. Data book says 'very strong beat every five waves'. (d) Note distortion of axial wave length. Data book says 'quite unsteady'. Flow changes from state $26 / 4$ to $26 / 5$ at $R=260$ (speed increasing). (e) Flow in process of changing from state $22 / 4$ to $22 / 5$ at nominal $R=679$. (f) Flow in process of changing from state $30 / 6$ to $28 / 5$ at nominal $R=1207$. Note reflexion of end disturbance in second cell.
(a) Photo. no. 145, $R=446$, state $18 / 6$.
(b) Photo. no. $44, R=1369$, state $24 / 5$.
(c) Photo. no. 124, $R=1314$, state $28 / 5$.
(d) Photo. no. $66, R=230$, state $26 / 4$.
(e) Photo. no. $129, R=675$, state changing from $22 / 4$ to $22 / 5$.
(f) Photo. no. 134, $R=1158$, state changing from $30 / 6$ to $28 / 5$. 

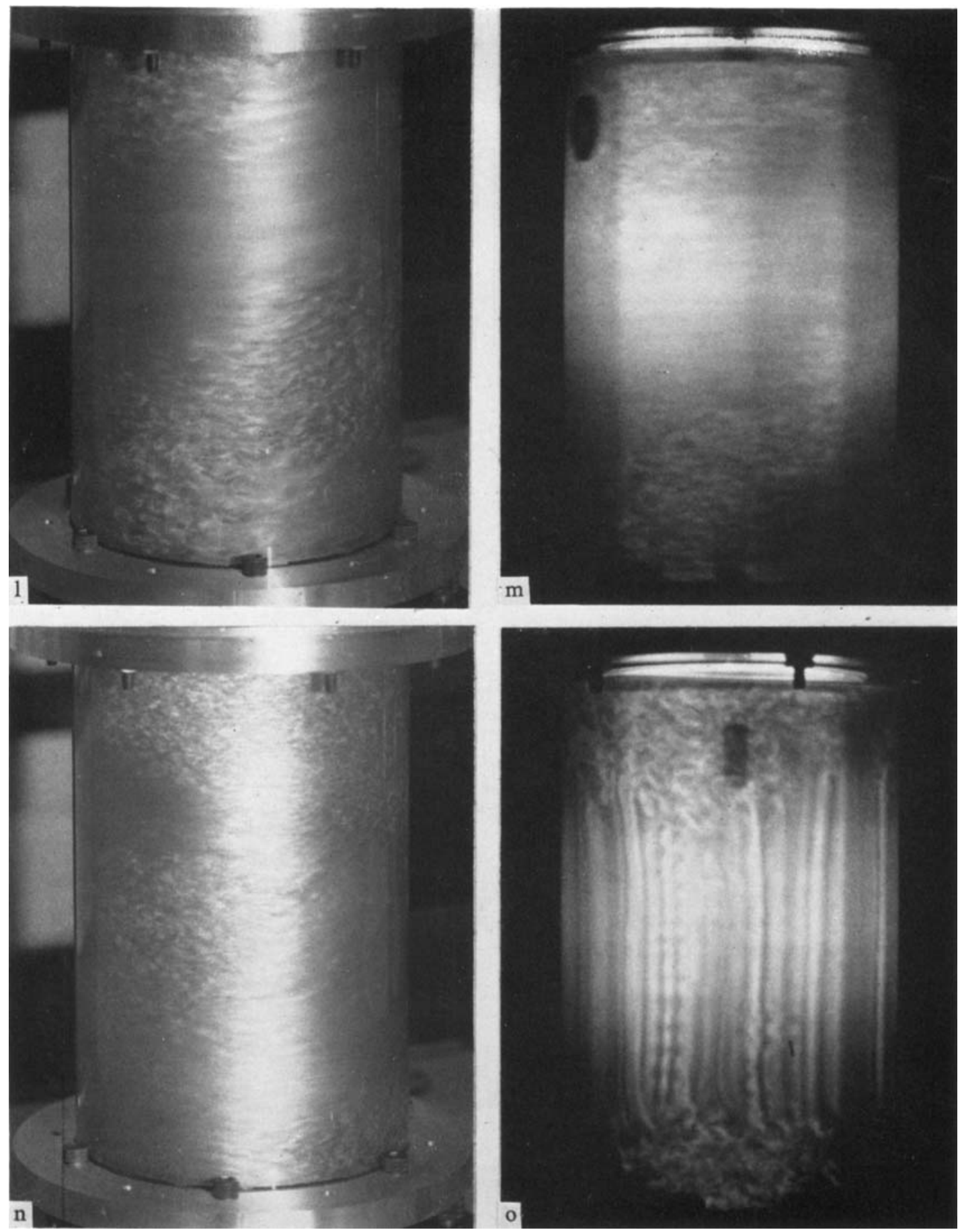

FrguRe 22. Intermittently turbulent flows, especially spiral turbulence, for opposite cylinder rotation (rough cylinders; $\left.r_{0} / r_{i}=1 \cdot 135\right)$. Light source for (l) and ( $\mathrm{n}$ ) is spark gap; scenes in $(\mathrm{m})$ and (o) are taken from motion picture. (l) Flow at $\mathrm{L}$ in figures $2(\mathrm{a})$ and (b); $R_{i}=5250, R_{0}=-15,880 .(\mathrm{m})$ Flow at $\mathrm{M}$ in figures $2(\mathrm{a})$ and $(\mathrm{b}) ; R_{i}=6210, R_{0}=-27,700$. (n) Flow at $\mathrm{N}$ in figure $2(\mathrm{a}) ; R_{i}=7280, R_{p}=-40,500$. (o) Instability following start-stop motion of outer cylinder.

COLES 
difference between $(a)$ the concept of an expected state, defined in the statistical sense; $(b)$ the concept of a preferred state, defined in the dynamic sense, and (c) the concept of a mean state, defined in terms of measurements like those in figure 10 .

\subsection{Some unfinished business}

An attempt has been made in figure 13 to collect some experimental and analytical material bearing on the uniqueness problem at relatively low speeds. As usual, the figure refers only to the simplest case of the inner cylinder rotating and the outer cylinder at rest. On the experimental side, the curve labelled 'expected state' is taken from table 1 and illustrates the observed tendency for the axial wave-number to decrease with increasing speed above the Taylor boundary. $\dagger$ A number of state transitions are again shown by arrows to emphasize the compulsive natural drive of the flow toward this expected or preferred state. On the analytical side, the main element is the Taylor boundary, together with an improvised second boundary through the experimental point

$$
R / R_{\text {crit. }}=1 \cdot 245, \quad a / a_{\text {crit }}=1 .
$$

One fundamental problem can now be simply stated. Consider a hypothetical steady Taylor flow at a higher speed than the first critical speed in figure 13, and suppose that the cylinders are infinitely long, or at any rate suppose that the axial constraints of a real experimental situation vanish, so that no one wavenumber or set of wave-numbers is distinguished a priori from any other. The question is: how is a unique wave-number to be determined, either analytically or experimentally, for such a flow? In particular, does the answer to this question have to be sought outside the framework of the Navier-Stokes equations?

Experimentally speaking, it is entirely feasible to avoid discrete changes in axial wave-number by continuously adjusting the length of a finite apparatus to the requirements of the motion for a fixed integral number of Taylor cells. However, for a given number of tangential waves and a given speed, there would still be some finite (not necessarily continuous) range of permissible lengths for the working space. $\ddagger$ Within this range, which would vary with noise level, the axial wave-number would not be uniquely determined by the experiment. Discrete and irreversible changes in tangential wave-number might also continue to occur at certain speeds in such an experiment, regardless of measures taken to prevent discrete changes in axial wave-number (a possible case in point is provided by

$\dagger$ Some support for these data is provided by the sequence of photographs published by Schultz-Grunow \& Hein (1956), in which there is a similar tendency for the axial wavenumber initially to decrease with increasing speed (Bilde $2,3,4,5$ ) up to a speed of about 20 times the first critical speed $\left(r_{o} / r_{i}=1 \cdot 19\right.$ for this apparatus). Without a knowledge of the operating history, of course, this information is not very useful.

\$ Some direct experimental evidence on this point has been obtained by Hagerty (1946) in a quite short apparatus with $r_{o} / r_{i}=1 \cdot 3$. For the case of the inner cylinder rotating at constant speed and the outer cylinder at rest, Hagerty found that the length of the working space could be varied by approximately a factor of two without a change in the number of Taylor cells. This same apparatus was apparently also operated over a wide range in speed at constant length without encountering the phenomenon of state transitions. Because of the relatively wide gap and short length of the apparatus, the role of the tangential instability in these observations is not clear. 
figure 12). The number of tangential waves is normally an integer, and a continuous variation in the tangential wave-number is not physically realistic unless the Taylor vortices have a spiral structure allowing a variation in phase between waves on adjacent vortex pairs. Such spiral configurations can be dealt with analytically, and they can also be realized experimentally, especially by a fast transit of the Taylor boundary when there is residual radial or tangential vorticity in the fluid. Except in the case of opposite rotation, however, these spiral motions are not permanent, at least in a short apparatus, and tend to change more or less rapidly to the usual configuration of closed vortex rings. All of these considerations make it doubtful that the question of uniqueness in a real experiment can ever be laid to rest by strictly experimental means.

The analytical methods recently developed by Stuart and Davey for dealing with the non-linear régime of Taylor flow have been mentioned in $§ 4.2$. These methods avoid the question of uniqueness by a 'shape assumption', in which the axial wave-number of the secondary motion is taken to be constant at the value given by the linearized solution at the Taylor boundary. $\dagger$ A less arbitrary assumption might be that the preferred wave-number, at least close to the Taylor boundary, is the one that is least stable in the sense of linearized theory. For flows of boundary-layer type, in which time and distance are nearly interchangeable variables, it is known that the linear stability theory is valid up to surprisingly large disturbance amplitudes, and that far downstream the particular frequency having the largest net amplification rate will tend to stand out against the general background because of selective amplification. To illustrate the corresponding situation for Couette flow, some analytical results due to Donnelly (private communication) have been included in figure 13 as the curve labelled 'maximum amplification'. This curve, which passes through the wavenumber having the largest amplification rate at each Reynolds number for the particular case $r_{o} / r_{i}=1 \cdot 111$, is evidently a poor approximation to the trajectory of the expected state in the figure.

Finally, a non-linear selection process having at least a superficial bearing on the state problem has been described by Segel (1962). This paper is a study of interaction between two disturbances (through non-linear coupling between two time-dependent amplitude equations of Landau-Stuart type) in flow due to thermal convection. The analysis again follows the precedent set by Stuart in taking the linearized problem as a point of departure, and the linearized amplifcation factors are again required to be small. Segel's conclusion for the case of two unstable disturbances having comparable wave-numbers and amplification factors is that the dominant one will be determined by the initial amplitudesalone.

In view of the important advances in non-linear technique which are contained in this analytical work by Stuart, Davey, Segel, and others, it is disappointing

$\dagger$ The shape assumption is sometimes justified by the remark that experimental evidence favours such a constant wave-number for the speed range where the non-linear theory should be valid. It would be more accurate to say that the speed of a finite apparatus can be slowly increased to a value well above the Taylor boundary (2.16 times the first critical speed in the present apparatus; probably less in a longer machine) before there is a transition leading to a change in the number of Taylor cells. 
that such analyses do not serve the present need; but this is unfortunately the case. The overriding difficulty, even for strictly laminar flow, is that the state problem extends to speeds so large that there is little hope of describing or measuring the effects of non-linearity by expansion procedures which are tied to linearized theory at the Taylor boundary and which emphasize the ideas of small amplitude and of conditions in the remote past. Whatever the speed, the experimentally observed state transitions have in common that one state or mode is initially present with a large amplitude, while other states are at most present as a kind of noise in the background. As the speed changes, however, one of these background states acquires the ability to extract energy from the original one and eventually to replace it. In many cases, this process of energy transfer manifests itself during the transition through a temporary inhomogeneity of the flow pattern. The motion depicted in figure 15 (d) (plate 2), for example, has 25 cells for part of the circumference and 27 cells for the remainder (this is a flow with a free surface). Which of the two competing states will eventually dominate depends on whether the partial vortex loop is increasing or decreasing in length. The motion depicted in figure 21 (e) (plate 11), on the other hand, has four waves at one end of the apparatus and five at the other. Again, which of the two competing states will eventually dominate depends on their relative strength in the no-man's-land near the centre of the apparatus.

A different and more attractive approach to the question of uniqueness has been proposed by Malkus \& Veronis (1958) for the problem of cellular convection in a fluid layer heated from below. These authors argue, after a brief investigation of the relative stability of alternative motions, that the preferred wave-number is determined by the operation of an extremum principle. The form of this principle is not entirely unambiguous, and there is also room for debate as to whether the principle should be treated as part of the content of the equations of motion or should be adduced separately. It is the latter view which will be adopted here. It may also be supposed, as a working hypothesis, that the extremum principle requires a maximum in the entropy production, or more practically a maximum in the torque for a given speed. This hypothesis has at least two virtues; it is not limited to any particular range of speed, and it fits well with the experimental fact that the possible flow patterns at a fixed speed are not uniformly or indifferently stable relative to each other. There does exist a preferred or expected state, which can be determined to a useful degree of precision by an empirical study of the dynamics of naturally occurring transitions. Before the evidence for such a preferred state is taken too seriously, however, some comments should be made about the completeness and credibility of the present experimental data.

In figure 10 , the first three sections, at $R=162,216,270$, give an impression of virtual completeness. These data correspond to the portion of the trajectory of the expected state included in figure 13, and the disagreement already noted with the trajectory for maximum amplification factor is therefore real. By the end of this range of Reynolds numbers, at approximately $\omega / \omega_{c}=2 \cdot 5$, the number of accessible states in figure 9 has gone through a first oscillation, and the expected state in figure 11 has settled down at roughly 22/6. 
The next few sections in figure 10, at $R=400-700$, correspond to an increase in the number of accessible states in figure $9 . \dagger$ They also strongly suggest a separation of the accessible region of wave-number space into two branches. One branch, centred roughly around the state $26 / 5$, is fairly well defined by the observations. The other branch, centred roughly around the state $16 / 3$, could not be observed in detail in these exploratory experiments, but its existence can be reliably inferred from the evidence of the various sections at $R=700-1300$ in figure 10. This branching process is also evident in the upper part of figure 12, where there are two stable regions of axial wave-number separated by an unstable region (cf. the situation for six waves at $R=864$ or 972 in figure 10). Whether or not the accessible region in wave-number space can become multiply connected at higher speeds is a moot point, inasmuch as the right (and presumably subordinate) branch in figure 10 could also be absorbed into the left branch by a series of transitions of the usual kind. $\ddagger$

To recapitulate, the conjecture at hand is that the trajectory of the expected state corresponds to a path of steepest ascent on a surface of dissipation rate plotted against speed and wave-number as independent variables. The surface belonging to the plane of figure 13, for example, might resemble the central part of the dissipation surface in figure 1 , provided that the co-ordinate $R_{o}$ in the latter is read as wave-number. Quite by accident, the shape of this surface in figure 1 is also relevant for a closer discussion of the phenomenon of branching. Due perhaps to some peculiarity of Wendt's experiment, there are two paths of steepest ascent on this surface, passing to right and left of a shallow couloir. One path follows roughly the line $R_{o}=0$, and the other follows roughly the line $R_{o}=-R_{i}$. On the assumption that the couloir terminates farther up on the surface, the two paths must eventually join each other at a branch point of the kind already postulated for the present state data.

The idea of maximum dissipation for the expected state can be investigated both analytically and experimentally, although there are formidable difficulties in both cases. Analytically, for example, the dependence of dissipation rate (or torque) on axial wave-number in a steady Taylor motion might be computed by variational methods $\S$ or by direct numerical integration of the Navier-Stokes

$\dagger$ In the speed range $0<R<1300$, it should be remembered, the spectrum for the doubly-periodic flows is still discrete; transition to turbulence has not yet officially begun (cf. plates 6 and 7). It is also unlikely that the noise originating at the ends of the apparatus for $R>690$ plays any decisive part in the state problem.

The hypothesis of branching is not contradicted by the experience of Schultz-Grunow \& Hein (1956). In their paper, Bilde 2-7 seem to indicate a trajectory along a high-wavenumber branch in the speed range up to about $\omega / \omega_{c}=100$. However, Bilde 8-9, in the range $100<\omega / \omega_{c}<200$, show a decrease in the axial wave-number by almost a factor of two, possibly indicating a transition to a different branch.

$\S$ This same variational problem might also be attacked experimentally. In the larger GALCIT apparatus, it is possible to vary the length of the working spece continuously while the cylinders are rotating, and thus to stretch or compress a given pattern of Taylor cells in the axial direction at fixed Reynolds number-at least within limits determined by the occurrence of state transitions in this machine. In precisely the sense of the variational calculus, therefore, the axial wave length might be varied at fixed Reynolds number, and an extremum sought in the dissipation. It was with such an experiment vaguely in mind, over and above the general need for probe traverses in the axial direction, that the 
equations, perhaps using an expansion method like that developed by Davey. The most direct evidence for the operation of an extremum principle, however, would be an experimental demonstration of a consistent discontinuity in torque during the various state transitions. An attempt has therefore been made to test this matter experimentally in the smaller GALCIT apparatus by measuring cylinder torque around several of the hysteresis loops mentioned in $\$ 4.3$. It should not matter that the torque so measured includes a large contribution from seal drag and from fluid friction outside the working space proper, inasmuch as the object is to detect differences in torque for different states at the same speed. Unfortunately, these measurements are quite difficult with the means available, and the only definite conclusion so far reached is that any change in torque must be quite small, of the order of $1 \%$ or less. An effort might be made to increase the contrast between adjacent states by decreasing the noise level, but in practice it is much easier to increase the noise level than to decrease it.

\section{Catastrophic transition}

\subsection{General survey}

From the standpoint of transition to turbulence, circular Couette flow between concentric rotating cylinders is similar in certain respects to flow in a pipe. For flows dominated by rotation of the inner cylinder, transition occurs through the process of slow spectral evolution associated with Taylor instability and described in \$4. For flows dominated by rotation of the outer cylinder, on the other hand, the experimental evidence shows that the basic circulatory motion is stable to infinitesimal disturbances, that the flow is, nevertheless, turbulent at sufficiently high speeds, and that there is an intermediate range of Reynolds numbers in which a stable mixed laminar-turbulent configuration can exist provided that sufficiently strong disturbances are present. These are the same properties which distinguish the pipe flow, the major difference being that any tendency toward periodicity of the intermittency phenomenon in the transition régime is reinforced in the case of Couette flow by the closed geometry.

It is true that the Taylor motion is sometimes a factor in catastrophic transition, but only as a source of finite disturbances. For example, consider the Taylor flow with opposite rotation depicted in figure 15(c) (plate 2), and suppose that the speed of the inner cylinder is increased. Depending on the speed of the outer cylinder, the next stage in the evolution of the flow is either a doublyperiodic flow with nearly stationary tangential waves in phase along the axis of rotation, or a partly turbulent flow with weak local transition occurring at random throughout the fluid. The two régimes are separated by a triple intersection on the second stability boundary at the extreme left in figure 2 (c). Beyond this intersection, which probably marks the closest approach of turbulence to the origin, an intermittency factor $\gamma$ may be defined in the usual way as the average fraction of the fluid participating in the turbulent motion, and the

movable end-plates were provided for this large apparatus. The measurements necessary for such a variational experiment, however, are of an almost incredible delicacy because of the low speeds involved. A more practical and equally elegant investigation might be made in the smaller machine by using a fluid-motion microscope or other tracer technique, except that this scheme is again almost unworkable in the doubly-periodic régime. 
new second boundary may be labelled $\gamma=0$. Slightly above this boundary, the main feature of the flow is a cyclic process which repeats itself indefinitely at constant speed. Small patches or spots of turbulence (with a turbulence scale comparable to the scale of the Taylor motion) appear, persist for a brief time, and decay, leaving the fluid calmed; the Taylor cells then reappear, grow in amplitude, and precipitate a fresh breakdown to turbulence. As the speed increases, the turbulent regions become larger and more viable, and the laminar regions become smaller and less viable, until finally, at the boundary labelled $\gamma=1$ in figure 2, laminar flow can no longer be detected. In general, the turbulence seems to be most completely developed and most homogeneous when the cylinders are rotating in opposite directions at nearly the same speed. The photographs figures $18(j)$ and $(k)$, in plate 5, for example, show two such flows at the points marked $J$ and $K$ in figure 2 (a). The decrease in turbulence scale with increasing speed is apparent. The sequence of photographs at the points $\mathrm{K}, \mathrm{I}, \mathrm{H}$ also illustrates the manner in which a dominant axial periodicity can emerge from the turbulent background as the operating conditions tend toward the case of the outer cylinder at rest.

For some distance in figure $2(b)$, the critical boundary for catastrophic instability lies slightly above the Taylor boundary. Toward the left side of the figure, however, the boundary $\gamma=0$ turns downward to cross the Taylor boundary, and beyond this intersection there is a region of hysteresis and nonuniqueness. $\dagger$ Below the line $\gamma=0$, turbulence if present will always decay if left to itself. Above the line $\gamma=0$, but below the next higher line in figure 2 (a) (a line consisting in part of a continuation of the Taylor boundary), turbulence if present will persist indefinitely, but it will not appear spontaneously if the flow is not disturbed. In this region, therefore, the Taylor boundary can still be observed if it is approached from the laminar side. In practice, of course, laminar flow cannot be maintained at arbitrarily high speeds, because unavoidable natural disturbances originating in the Taylor motion, in the vorticity anomaly at the ends of the working space, in the wake behind a probe, in mechanical vibration, and so on, will sooner or later trigger the transition to turbulence. Artificial disturbances such as an air jet can also be used to trip the flow in the hysteresis region. Once turbulence has appeared, for whatever reason, this turbulence takes pre-emptive control over the flow situation, and the laminar state can only be restored by a period of operation on the laminar side of the boundary $\gamma=0$.

A reasonable interpretation of the hysteresis phenomenon is that operating conditions along the line $\gamma=0$ correspond to an energy threshold. In the vicinity of this threshold, transition to turbulence can be caused by any one of a variety of finite disturbances. The local energy delivery to the fluid at the moving walls then increases in direct proportion to the increase in wall shearing stress due to the turbulence.' Provided that the operating point is above the boundary $\gamma=0$, the increased energy supply can satisfy the increased demand, and thus maintain

+ Two different fluids were used to obtain the data shown in figure $2(a)$. The reason for the discrepancy in the position of the boundary $\gamma=0$ for the two fluids is not known; it is possible that the apparatus was not completely filled with fluid in one cese or the other. 
the turbulence, even if the disturbances are removed. The interpretation of the intermittency, however, is more difficult. If there were three dimensions for the volume of turbulent fluid and only two dimensions for the energy source at the moving walls, then an increase in the turbulent volume at constant speed would not necessarily call up a corresponding increase in the rate of energy supply. The operation of such a principle is by no means obvious, however, and an investigation of the remarkable stability of these mixed laminar-turbulent flows presents a real experimental challenge.

\subsection{Spiral turbulence}

Among the various intermittently turbulent flows which can occur in the region of catastrophic transition, there is one quite common and at the same time quite special configuration. Two photographs of this flow, at the points $L$ and $M$ respectively in figure 2 , are shown in figures $22(1)$ and $(\mathrm{m})$ (plate 12). The flow consists of a spiral band of turbulence, either right-hand or left-hand, which rotates steadily at essentially the mean angular velocity of the two cylinders without changing its shape or losing its identity. This spiral configuration has been observed in flow with the inner cylinder at rest and even in flow with the two cylinders rotating in the same direction. In general, the spiral pattern is most regular at the lower speeds in the central part of the transition region in figure 2. Near either of the transition boundaries $\gamma=0$ or $\gamma=1$, there is increasing randomness and degeneracy of the spiral structure, as illustrated in figure $22(\mathrm{n})$ (plate 12), for the flow at the point $\mathrm{N}$ in figure 2 (a). Preliminary hotwire measurements in the large GALCIT apparatus by H. Oguro and C. Van Atta have also shown that the intermittency factor $\gamma$ is relatively greater near the centre of the annular gap than near the walls. In some extreme cases, the flow at mid-radius can be fully turbulent even though laminar flow is still present near one or both walls. Finally, although the normal configuration is a single spiral, one case of a second mode having a double spiral structure has been observed by H. Oguro.

Hot-wire data obtained in these spiral turbulent flows indicate that the fluid moves on the average in nearly circular paths, so that a fluid element near either wall repeatedly traverses the spiral pattern and hence participates alternately in the laminar and turbulent motions. It follows that there must be two kinds of interfaces separating regions of laminar and turbulent flow. One kind represents transition in the normal sense, with laminar regions becoming turbulent; the other kind represents inverse or anti-transition, with turbulent regions becoming laminar. The two kinds of interface appear to be almost equally sharply defined and to propagate at comparable speeds with respect to the fluid. Such interfaces have been observed previously in other flows, including the pipe and channel flow and the boundary layer. It is probably more than coincidence that all of these flows are constrained by walls and that there is a relatively high level of vorticity in the ambient laminar flow. The experimental conditions for these flows are rather awkward, however, in that the intermittency tends to be quite irregular unless it is artificially controlled. In the case of Couette flow, on the other hand, the inherent regularity of the spiral turbulence makes it an ideal 
target of opportunity for an investigation of interfaces and intermittency. In fact, the spiral flow in a rotating reference frame may be viewed as an isolated region of captive turbulence in equilibrium with its environment. This region remains essentially fixed in size and shape even though there is a continuous exchange of fluid with the surrounding laminar regions, an exchange which is similar to the one noted in $\$ 4.3$ for the large-eddy motions represented by tangential waves in doubly-periodic laminar flow. In the case of spiral turbulence this exchange process implies a continuous catastrophic instability all along the transition interfaces, and a statement about interface propagation is likely also to be a statement about instability to finite disturbances.

The main problem in connexion with intermittency is to identify the mechanism which accounts for the disappearance of turbulence in certain regions of the fluid, and more generally to study the factors which control the rate and direction of interface propagation. The observed sharpness of the anti-transition interfaces raises the possibility that the anti-transition mechanism is not entirely diffusive or viscous in nature. In other words, part of the turbulent energy may actually be transferred from the turbulence back to the mean flow rather than merely dissipated as heat. The mechanism which suggests itself is cancellation of vorticity, by a process inverse to the one which originally produced the same vorticity in another part of the flow. The idea of vorticity cancellation, or unstretching, by a reversible non-diffusive process was also encountered in $\S 4$ of this paper in connexion with state transitions involving a decrease in the axial wave-number of a periodic laminar flow. This idea is also related to the idea of energy transfer from smaller to larger eddies in any turbulent motion. At the same time, the Reynolds number in flows with anti-transition is usually small enough so that at least the large-scale part of the vorticity structure may always retain a fairly high degree of organization.

To investigate these questions, an elaborate experiment is being carried out in the large GALCIT apparatus by C. Van Atta and the author. This experiment consists of repeated digital sampling of the instantaneous velocity vector at various points fixed with respect to the rotating spiral pattern. At each such point the mean velocity, the Reynolds stresses, and the mean rate of turbulent energy production are to be determined as ensemble averages over a large number of such samples. The crucial issue is the algebraic sign of the turbulence production: is this quantity positive definite, like its companion the viscous dissipation, or can it be negative, indicating transfer of energy from the turbulence to the mean flow? This experiment is well advanced at the present writing, and there is every reason to believe that the desired quantities have been successfully measured. However, the result of the experiment is not yet known.

The experimental research reported in this paper was supported from 1955 to 1961 by the National Science Foundation, and from 1961 to 1963 by the Jet Propulsion Laboratory. This support is gratefully acknowledged. Preparation of the manuscript and figures was aided by funds made available by G. Carrier in connexion with contract Nonr-1866(20). Some of the results have previously been reported at a meeting of the Fluid Dynamics Division of the American 
Physical Society in San Diego in 1958, at the Tenth International Congress for Applied Mechanies in Stresa, Italy, in 1960, and at an International Colloquium on Turbulence in Marseilles, France, in 1961.

\section{REFERENCES}

Covette, M. 1890 Ann. Chim. Phys. (6), 21, 433-510.

DAveY, A. 1962 J. Fluid Mech. 14, 336-68.

DiPrima, R. C. 1961 Phys. Fluids, 4, 751-5.

DonNelly, R. J. 1963 Phys. Rev. Letters, 10, 282-4.

Fraenkel, L. E. 1956 Proc. Roy. Soc. A, 233, 506-26.

FuLtz, D. 1961 Adv. Geophys. 7, 1-103.

GöRTLER, H. $1944 Z$. angew. Math. Mech. 24, 210-14.

Hagerty, W. W. 1946 Ph.D. dissertation, University of Michigan, Ann Arbor.

KovasZday, L. S. G. 1949 Proc. Heat Transf. Fluid Mech. Inst. 211-22.

LEWIs, J. W. 1928 Proc. Roy. Soc. A, 117, 388-406.

MaLKUS, W. V. R. \& Veronrs, G. 1958 J. Fluid Mech. 4, 225.

MALLOCK, A. 1888 Proc. Roy. Soc. A, 45, 126-32.

Mallock, A. 1896 Phil. Trans. A, 187, 41-56.

PAI, S.-I. 1943 NACA TN 892.

RAYLeIgH, LoRD 1916 Proc. Roy. Soc. A, 93, 148-54.

Roshko, A. 1955 NACA TN 3488.

Schultz-Grunow, F. \& Hein, H. 1956 Z. Flugwiss. 4, 28-30.

Stged, L. A. $1962 J$. Fluid Mech. 14, 97.

StUART, J. T. 1958 J. Fluid Mech. 4, 1-21.

TAYLOR, G. I. 1923 Phil. Trans. A, 223, 289-343.

TAYLOR, G. I. 1936 Proc. Roy. Soc. A, 157, 546-64.

Terada, T. \& Hattori, K. 1926 Rep. Tokyo Aeron. Res. Inst. 2 (no. 26), 287-326.

WENDT, F. 1933 Ing. Arch. 4, 577-95. 